\title{
An immersed boundary-lattice Boltzmann method for fluid-structure interaction problems involving viscoelastic fluids and complex geometries
}

\author{
Jingtao Ma ${ }^{\mathrm{a}}$, Zhen Wang ${ }^{\mathrm{b}}$, John Young ${ }^{\mathrm{a}}$, Joseph C.S. Lai ${ }^{\mathrm{a}}$, Yi Sui ${ }^{\mathrm{b}}$, Fang-Bao Tian ${ }^{1 \mathrm{a}}$ \\ ${ }^{a}$ School of Engineering and Information Technology, University of New South Wales, Canberra, ACT 2600, \\ Australia \\ ${ }^{b}$ School of Engineering and Materials Science, Queen Mary University of London, London E1 4NS, UK
}

\begin{abstract}
An immersed boundary-lattice Boltzmann method (IB-LBM) for fluid-structure interaction (FSI) problems involving viscoelastic fluids and complex geometries is presented in this paper. In this method, the fluid dynamics and the constitutive equations of viscoelastic fluids are both solved using the lattice Boltzmann method. In order to enhance numerical stability in solving the constitutive equations, an artificial damping is introduced which does not affect the numerical results if the damping effect is much smaller than the relaxation and the convective effects. The structural dynamics including 2D and 3D capsules, 2D and 3D rigid particles and flags, are solved by the finite difference method (2D capsules, 2D and 3D rigid particles and flags) and the finite element method (3D capsules). The interaction between the solid structure and the fluid is enforced by an immersed boundary method. The overall framework of this method is very simple, enabling modelling FSI problems involving viscoelastic fluids and the inertia of both fluids and structures. It is very efficient for FSI problems involving high Weissenberg numbers flows, large deformations and complicated geometries without any preconditioner. This work uses IB-LBM to solve for the first time, flows involving viscoelastic fluids coupled with non-massless deforming structures. The method is also capable of solving very high Weissenberg number problems, as demonstrated by simulations of flexible particle flows at $W i=100$. The present method and models are validated by several cases including a 2D rigid particle migration in a Giesekus Couette flow, a spherical particle rotation in an Oldroyd-B shear flow, a spherical particle settling in a FENE-CR fluid, 2D and 3D capsules deformation in a Newtonian shear flow, and a 3D flag flapping in a Newtonian free stream. In addition, the present method is also applied to simulate the deformation of $2 \mathrm{D}$ and $3 \mathrm{D}$ capsules in an Oldroyd-B shear flow, a 3D flag flapping in an Oldroyd-B free stream, and elastic capsule movement in a contraction-expansion channel filled with an Oldroyd-B fluid. Deformation of the capsules decreases with the increase of the Weissenberg number and the capsules experience monotonically increasing deformation when the Weissenberg number is above a critical value which is respectively 10 for $2 \mathrm{D}$ and 2 for $3 \mathrm{D}$ simulations. Viscoelasticity of the Oldroyd-B fluid hinders the flapping motion of the 3D flag. For elastic capsules passing through a periodic contractionexpansion channel, the capsules mix up after a short-term evolution, and then migrate to the bottom of the channel and almost follow two steady trajectories after a long-term evolution. The validations and applications provide extensive data which may be used to expand the currently limited database available for FSI benchmark studies.
\end{abstract}

Key words:

\footnotetext{
${ }^{1}$ Correspondence author. Email: f.tian@adfa.edu.au; onetfbao@gmail.com.
} 
Immersed-boundary method; lattice Boltzmann method; fluid-structure interaction; viscoelastic fluids

\section{Introduction}

Non-Newtonian fluid behaviours (e.g., shear-thinning, shear-thickening, yield-stress, viscoelastic and viscoplastic) are involved in many industrial processes and biological systems, such as polymer and melt solutions, pulp and paper, minerals, food, pastes and cosmetics processing, and mucus over fish and biological internal lumen. Specifically, the mucus layer covering the luminal surface of the airway is a type of viscoelastic fluid and plays an important role in the mucociliary clearance system, trapping the inhaled external particles and assisting the beating cilium to transport the particles out of the lung [1]. Compared with Newtonian fluids, non-Newtonian fluids exhibit several so called "anomalous" behaviours [2]. For example, turbulence may appear at extremely small Reynolds numbers (e.g., $R e \approx 10^{-3}[3]$ ) in viscoelastic fluids, in contrast to high Reynolds numbers in Newtonian flow. Non-Newtonian flows can also lead to different solid particle behaviours when the fluid-structure interaction (FSI) is considered. For example, in Newtonian fluids, a repulsion between the particle and the wall is normally produced by inertia when a spherical particle drops close to a vertical wall [4], however in viscoelastic fluids the particle can be pushed towards the wall [4]. In many industrial processes, non-Newtonian rheology can be used to improve the designs. For example, recently viscoelastic fluids have been used to separate different particles $[5,6,7]$, cells [8], and bacteria [9]. Studies show that it can be more effective to manipulate particles in non-Newtonian fluids than in Newtonian fluids $[10,11,12]$. Recent progress on particle motion in non-Newtonian fluids has been summarized in reviews by Lu et al. [10], Yuan et al. [13] and D'Avino et al. [14].

To understand the behaviours of solid structures in viscoelastic fluids, experimental and numerical methods have been employed $[2,5,6,15,16,17,18,19,20,21,22,23,24]$. In experimental studies, Becker et al. [15] investigated spherical particle sedimentation in a bounded cylindrical tube filled with viscoelastic fluids, and found that the particle velocity experiences an overshoot of up to $50 \%$ before approaching the ultimate steady value. Bodart and Crochet [16] reported that the magnitude of the initial overshoot depends on several factors, such as the Weissenberg number, particle density, solvent viscosity, and wall effects. Rigid particle separation in viscoelastic fluids has also been studied experimentally $[17,5,6]$.

Over the last several decades, numerical methods have also become prevalent in the research of FSI problems involving viscoelastic fluids [2, 18, 19, 20, 21, 22, 23, 24, 25]. Numerical simulation of the solid structure motion in viscoelastic fluids is challenging, particularly at high Weissenberg number. Hulsen et al. [26] reported that the finite element method without stabilization for a viscoelastic flow over a cylinder did not converge for Weissenberg number beyond a critical value of approximately 1.0. Different strategies have been proposed to stabilize the solution for viscoelastic fluids, such as log-conformation representation (LCR) by Fattal and Kupferman [27], and squareroot-conformation representation (SRCR) by Balci et al. [28]. The LCR and SRCR methods are able to improve the stability of the numerical simulation at high Weissenberg numbers (e.g., Wi $>1.0$ for the Oldroyd-B flow over a cylinder and $W i>5.0$ for Oldroyd-B flow in a 2D lid-driven cavity) $[26,29]$, but these methods may increase the computation cost as well [29].

Some early attempts to model FSI problems involving non-Newtonian flows were based on body conformal meshes, such as the arbitrary Lagrangian Eulerian-finite element method (ALE-FEM) 
$[2,30,31,32,33,34]$ and deforming-spatial-domain/stabilized space-time (DSD/SST) method $[35,36,37,38,39,40]$. For example, Feng and his collaborators have utilized ALE-FEM to investigate rigid particle, bubble and drop movement in viscoelastic fluids [2, 30, 33, 34]. The ALE-FEM method has also been used to study elastic particle behaviours in viscoelastic fluids [31, 32]. In these methods, meshes are generated to conform to the instantaneous shapes of the immersed body, move with the moving boundaries, and generally need to be regenerated when large deformation/displacement is involved. As an alternative, methods based on non-conformal meshes are more convenient regarding ease and robustness of mesh implementation. Some of these are the distributed Lagrange multiplier/fictitious domain method (DLM/FDM) [19, 41, 42] and the immersed boundary method (IBM) $[43,44,45]$, the latter of which was initially developed to model the blood flow in the human heart by Peskin [46, 47]. In IBM, a fixed Cartesian mesh is used to describe the fluid and the immersed boundary is represented by a set of Lagrangian points. A force density is spread onto the ambient fluid to account for the effect of the boundary. Peng and Luo [45] have utilized IBM to study the 2D flow over a circular cylinder, Kim and co-workers used IBM to study rigid particle and deformable vesicle behaviours in viscoelastic fluids [43, 44], and DLM/FDM was employed to simulate rigid particle behaviours in Oldroyd-B fluids by Pan and his collaborators $[41,42]$.

The flow solvers in the above numerical studies are directly based on the Navier-Stokes equations, which normally handle nonlinear operators and pressure Poisson equations. An alternative and robust method for fluid flow simulation is the lattice Boltzmann method (LBM), which has gained growing popularity in recent years due to its simple formulation and high level of scalability on parallel processing systems. The LBM is an approach based on particle kinetics, avoiding discretising the Navier-Stokes equations [48, 49]. To combine the advantages of IBM and LBM, they have been coupled to investigate FSI problems in Newtonian and power-law non-Newtonian fluids $[50,51,52,53,54,55,56,57,58,59,60,61,62,63,64,65]$.

Recently, LBM has also been used to study FSI problems in viscoelastic fluids [21, 24, 66]. Goyal and Derksen [21] utilized the LBM to describe the flow of the fluids, the finite volume method was employed to solve the constitutive equations of viscoelastic rheology, and the IBM was used to model the FSI. This method was validated with a series of benchmark cases including 2D Oldroyd-B flow over a cylinder, a 3D spherical particle rotation in an Oldroyd-B shear flow, and a 3D spherical particle sedimentation in Newtonian fluid under gravity. More recently, Lee and Ahn [24] developed a LBM-smoothed profile method (SPM) method for viscoelastic suspensions. In this method, the LBM originally proposed by Malaspinas et al. [67] was used to solve the viscoelastic flow, and the SPM was employed to calculate the rigid particle dynamics. This method was able to solve viscoelastic flows of Weissenberg number up to 1.6 for flow over a cylinder without a stabilization scheme. The LBM was coupled with IBM by Zhu [66] to investigate 3D FSI of flows over a massless flexible sheet. It was found that the viscoelasticity of the fluid inhibited three-dimensionality in the flag motion for the cases considered. Despite the above studies, FSI problems involving viscoelastic fluids and the inertia of both fluids and structures have not been considered. In addition, effort is required to extend the IB-LBM to FSI problems involving high Weissenberg number flows, large deformations and complicated geometries. The objective of this work is to expand the application of the IB-LBM and to extend the current limited data base of FSI problems.

In this work, we present an immersed boundary-lattice Boltzmann method for FSI problems involving viscoelastic fluids and complex geometries. The Navier-Stokes equations and constitutive equations of viscoelastic models are solved by using the LBM developed by Malaspinas et al. [67] . In 
order to enhance the numerical stability in solving the constitutive equations, an artificial damping is introduced. This treatment is very simple, but is able to extend the simulation capability of IB-LBMfor the first time to FSI problems involving both non-massless deforming structures and fluid inertia (i.e. higher Reynolds number) as well as at high Weissenberg numbers (e.g., $\mathrm{O}\left(10^{2}\right)$ ) without any preconditioner. The structure dynamics including 2D and 3D capsules, 2D and 3D rigid particles and flags are solved by the finite difference method (2D capsules, 2D and 3D rigid particles, and flags) and the finite element method (3D capsules). Interaction between the moving surface and the fluid is accomplished with the IBM.

The organisation of the rest of this paper is as follows. The governing equations of the fluid dynamics and solid structures are introduced in Section 2. Section 3 presents the numerical approach. In Section 4, several validation cases are presented. Section 5 gives several applications of the present method. Final conclusions are provided in Section 6 .

\section{The mathematical model}

In this work, we consider incompressible viscoelastic fluids (e.g., an aqueous polymer solution) and several structures including 2D and 3D capsules, 2D and 3D rigid particles and flags. The mathematical models used to describe the dynamics of the fluid and the solid structures are introduced in this section.

\subsection{The governing equations of the fluid dynamics}

The dynamics of an incompressible fluid are described by the continuity and Navier-Stokes equations,

$$
\begin{gathered}
\nabla \cdot \boldsymbol{u}=0 \\
\frac{\partial \boldsymbol{u}}{\partial t}+(\boldsymbol{u} \cdot \nabla) \boldsymbol{u}=\frac{1}{\rho} \nabla \cdot\left(-p \boldsymbol{I}+2 \mu_{s} \boldsymbol{D}+\boldsymbol{\tau}_{\boldsymbol{p}}\right),
\end{gathered}
$$

where $\rho$ is the fluid density, $\boldsymbol{u}$ is the fluid velocity, $p$ is the pressure, $\mu_{s}$ is the solvent dynamic viscosity, $\boldsymbol{I}$ is the identity tensor, $\boldsymbol{D}=\frac{1}{2}\left(\nabla \boldsymbol{u}+(\nabla \boldsymbol{u})^{T}\right)$ is the strain rate tensor, and $\boldsymbol{\tau}_{p}$ is the viscoelastic stress tensor which accounts for the effect of the polymers on the solvent. There are several models which are used to determine $\tau_{p}$ such as Oldroyd-B, FENE-CR and Giesekus $[67,21,24]$. In these models, the viscoelastic stress tensor $\tau_{p}$ can be described by the conformation tensor $\boldsymbol{C}$ (a statistical indicator of the orientation of the polymer molecules) [67, 21],

$$
\begin{gathered}
\tau_{\boldsymbol{p}}=\frac{\mu_{p}}{\lambda}(\boldsymbol{C}-\boldsymbol{I}) \quad(\text { Oldroyd }-\mathrm{B} \text { and Giesekus }), \\
\boldsymbol{\tau}_{\boldsymbol{p}}=\frac{\mu_{p}}{\lambda} F(\boldsymbol{C})(\boldsymbol{C}-\boldsymbol{I}) \quad(\text { FENE }-\mathrm{CR}),
\end{gathered}
$$

where $\mu_{p}$ and $\lambda$ are respectively the dynamic viscosity and relaxation time of the polymer, and $F(\boldsymbol{C})=\frac{1}{1-\operatorname{tr}(\boldsymbol{C}) / L_{p}^{2}}$ is the spring function with $L_{p}$ being the maximum extension length of the spring and $\operatorname{tr}(\boldsymbol{C})$ being the trace of the tensor $\boldsymbol{C}$. The conformation tensor $\boldsymbol{C}$ is determined by the following transport equations,

$$
\frac{\partial \boldsymbol{C}}{\partial t}+(\boldsymbol{u} \cdot \nabla) \boldsymbol{C}=-\frac{1}{\lambda}(\boldsymbol{C}-\boldsymbol{I})-\frac{\alpha}{\lambda}(\boldsymbol{C}-\boldsymbol{I}) \cdot(\boldsymbol{C}-\boldsymbol{I})+\boldsymbol{C} \cdot \nabla \boldsymbol{u}+(\nabla \boldsymbol{u})^{T} \cdot \boldsymbol{C} \quad \text { (Giesekus), }
$$




$$
\frac{\partial \boldsymbol{C}}{\partial t}+(\boldsymbol{u} \cdot \nabla) \boldsymbol{C}=-\frac{F(\boldsymbol{C})}{\lambda}(\boldsymbol{C}-\boldsymbol{I})+\boldsymbol{C} \cdot \nabla \boldsymbol{u}+(\nabla \boldsymbol{u})^{T} \cdot \boldsymbol{C} \quad(\mathrm{FENE}-\mathrm{CR}),
$$

where $\alpha$ is the mobility parameter. The Oldroyd-B model can be recovered from the Giesekus model for the case of $\alpha=0$ and FENE-CR model for the case of $L_{p}^{2} \rightarrow \infty$.

\subsection{The solid structure models}

In this work, several solid structures including deformable capsules, rigid particles, and a flexible flag are considered. The governing equations of these structures are given below.

\subsubsection{The 2D deformable capsule model}

The membrane of the 2D capsule is treated as a zero-thickness elastic surface. The schematic geometry of a 2D capsule is illustrated in Fig. 1. The unit tangent vector $\mathbf{t}$ points to the direction of increasing arc length $(s)$, and the normal unit vector $\mathbf{n}$ points to the outer fluid. Due to the deformation of the capsule, the membrane develops an in-plane tension $\tau$ and a transverse shear tension $q$ which are associated with the stretching and bending deformations. Assuming that the mass of the capsule membrane is ignored, a force balance requires

$$
\boldsymbol{F}_{H}=-\frac{d}{d s}(\tau \mathbf{t})+\boldsymbol{f}_{b},
$$

where $\boldsymbol{F}_{H}$ is the hydrodynamic force on the membrane, and $\boldsymbol{f}_{b}$ is the elastic force due to bending deformation. Here the Hooke's law is used to evaluate the in-plane tension $\tau$, i.e.

$$
\tau=k_{s}\left(\left|\frac{\partial \mathbf{X}}{\partial s_{0}}\right|-1\right)
$$

where $k_{s}$ is the stretching coefficient of the capsule membrane, $\mathbf{X}$ is the position vector of a point on the capsule membrane, and $s_{0}$ is the initial arc length. The bending component $f_{b}$ of the interfacial tension is computed as [68],

$$
\boldsymbol{f}_{b}=-\frac{d}{d s}(q \mathbf{n}),
$$

where $q$ is expressed in terms of the bending moment $M$ as follows,

$$
q=\frac{d M}{d s}
$$

and the bending moment is computed by

$$
M=k_{b}\left[\epsilon(s)-\epsilon_{0}(s)\right],
$$

where $\epsilon(s)$ is the instantaneous membrane curvature, and $\epsilon_{0}(s)$ is the initial curvature of the membrane at minimum bending energy configuration [68, 69]. 


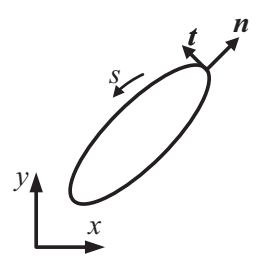

Figure 1: The schematic geometry of $2 \mathrm{D}$ capsule.

\subsubsection{The 3D deformable capsule model}

Similar to the 2D capsule, the membrane of the 3D capsule is treated as a zero-thickness elastic surface. As it is not easy to develop the 3D capsule dynamics using the Cartesian system, here we introduce the 3D capsule model from an energy point of view. The elastic and bending energy may be produced due to the deformation of the capsule. In this work, we consider two capsule models which are respectively based on the neo-Hookean and Skalak's law. According to the neo-Hookean (NH) law which was initially developed to model the volume-incompressible rubber-like materials, the strain energy due to the stretching deformation takes the form of $[70,51]$

$$
W^{S}=\int_{A} \frac{E_{s}}{6}\left(I_{1}+\frac{1}{I_{2}+1}-1\right) d A
$$

where $W^{S}$ is the total strain energy density, $A$ is the surface area, $E_{s}$ is the surface shear elasticity modulus, and $I_{1}$ and $I_{2}$ are the strain invariants of the surface deformation, calculated by the principal in-plane stretch ratios $\lambda_{1}$ and $\lambda_{2}$ according to

$$
\begin{aligned}
& I_{1}=\lambda_{1}^{2}+\lambda_{2}^{2}-2, \\
& I_{2}=\left(\lambda_{1} \lambda_{2}\right)^{2}-1 .
\end{aligned}
$$

Another model is based on the Skalak's (SK) law, which was introduced by Skalak et al. [71] to model the membrane of the red blood cells (RBC). In this model, the strain energy function due to the stretching deformation is given by

$$
W^{S}=\int_{A}\left[\frac{E_{s}}{12}\left(I_{1}^{2}+2 I_{1}-2 I_{2}\right)+\frac{E_{a}}{12} I_{2}^{2}\right] d A
$$

where $E_{a}$ is elastic modulus for area dilation for surface-area conservation, and the RBC should have a large value $\left(O\left(10^{5}\right)\right)$ of the ratio of the area dilation to elasticity modulus $C=E_{a} / E_{s}$ to ensure that the change in the surface area is negligible [72]. It should be noted that the SK law is equivalent to the $\mathrm{NH}$ law in the small deformation regime when $C=1$.

Here, the membrane bending resistance is described by the Helfrich bending energy [73, 74],

$$
W^{B}=\frac{E_{b}}{2} \int_{A}\left(2 H-c_{0}\right)^{2} d A,
$$

where $W^{B}$ is the capsule membrane bending energy, $E_{b}$ is the bending rigidity, $H$ is the mean curvature, and $c_{0}$ is the curvature of the capsule membrane at the rest state. Here, following the research by Wang et al. [51], $c_{0}=0$ is used.

Finally, a force balance is achieved, 


$$
\boldsymbol{F}_{H}=-\left(\boldsymbol{f}_{e}+\boldsymbol{f}_{b}\right)=\frac{\delta\left(W^{S}+W^{B}\right)}{\delta \mathbf{X}},
$$

where $\boldsymbol{f}_{e}$ and $\boldsymbol{f}_{b}$ are respectively the internal elastic and bending forces induced by the deformation of the membrane.

\subsubsection{The rigid particle equations}

The motion of the rigid particle follows Newton's second law,

$$
\begin{aligned}
& m \frac{d \boldsymbol{V}}{d t}=\boldsymbol{F}_{e x t}, \\
& {[I] \frac{d \boldsymbol{\Omega}}{d t}=\boldsymbol{T}_{\text {ext }},}
\end{aligned}
$$

where $m$ and $[I]$ are respectively the mass and moment of inertia of the particle, $\boldsymbol{V}$ and $\boldsymbol{\Omega}$ are respectively the translational and rotational velocity of the particle, and $\boldsymbol{F}_{\text {ext }}$ and $\boldsymbol{T}_{\text {ext }}$ are respectively the external force and moment acting on the particle including the hydrodynamic force, the gravity force (if any) and any other additional force depending on the problem.

\subsubsection{The governing equation of the flexible flag}

For the flag which is a rectangular flexible plate, it is convenient to write the governing equation in a curvilinear coordinate system [75],

$$
\rho_{s} \frac{\partial^{2} \mathbf{X}}{\partial t^{2}}=\sum_{i, j=1}^{2}\left[\frac{\partial}{\partial s_{i}}\left(\sigma_{i j} \frac{\partial \mathbf{X}}{\partial s_{j}}\right)-\frac{\partial^{2}}{\partial s_{i} \partial s_{j}}\left(\kappa_{i j}^{B} \frac{\partial^{2} \mathbf{X}}{\partial s_{i} \partial s_{j}}\right)\right]+\boldsymbol{F}_{H},
$$

where $\rho_{s}$ denotes the area density of the flag, $\sigma_{i j}=\kappa_{i j}^{T}\left(\frac{\partial \mathbf{X}}{\partial s_{i}} \cdot \frac{\partial \mathbf{X}}{\partial s_{j}}-T_{i j}^{0}\right), \boldsymbol{F}_{H}$ is the hydrodynamic force exerted on the flag by the ambient fluid, $\boldsymbol{X}$ is the position of the flag, and $\left(s_{1}, s_{2}\right)$ are the curvilinear coordinates along and across the flag. $\kappa_{i j}^{T}$ is the tension $(i=j)$ or shearing $(i \neq j)$ coefficient, $\kappa_{i j}^{B}$ is the bending $(i=j)$ or twisting $(i \neq j)$ coefficient, and $T_{i j}^{0}$ denotes the initial stretching ratio. For initially unstretched case, $T_{i j}^{0}$ is given by,

$$
T_{i j}^{0}=\left\{\begin{array}{l}
1, \text { if } i=j, \\
0, \text { if } i \neq j .
\end{array}\right.
$$

\section{Numerical approach}

In this section, the numerical approaches used to solve the governing equations of the fluid and the solid structures are introduced. The Navier-Stokes equations and constitutive equations of the viscoelastic models are solved by using the LBM, and the governing equations of the solid structures are solved by the finite difference method (2D capsules, 2D and 3D rigid particles and flags) and finite element method (3D capsules). 
(a)

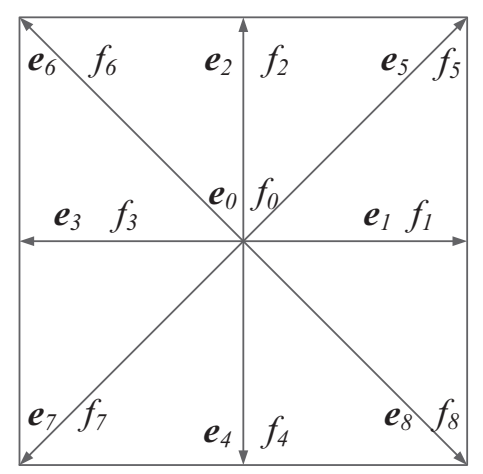

(b)

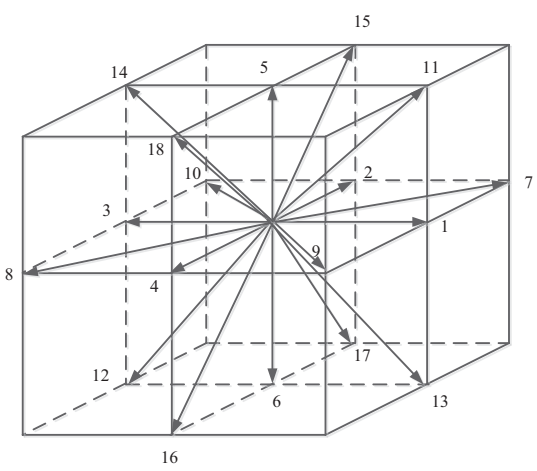

Figure 2: (a) D2Q9 lattice model. (b) D3Q19 lattice model.

\subsection{The lattice Boltzmann method for the governing equations of the fluid}

\subsubsection{The lattice Boltzmann method for the Navier-Stokes equations}

The discrete lattice Boltzmann equation of a single relaxation time model is utilized to solve the Navier-Stokes equations where $\frac{1}{\rho} \nabla \cdot \tau_{\boldsymbol{p}}$ (in Eq. 2) is taken as external forces [48, 52]

$$
f_{i}\left(\mathbf{x}+\mathbf{e}_{i} \Delta t, t+\Delta t\right)-f_{i}(\mathbf{x}, t)=-\frac{1}{\tau}\left[f_{i}(\mathbf{x}, t)-f_{i}^{e q}(\mathbf{x}, t)\right]+\Delta t F_{i},
$$

where $f_{i}$ is the density distribution function, $t$ is the time, $\mathbf{x}$ is the fluid parcel position, $\mathbf{e}_{i}$ is the discrete velocity along the $i$ th direction, $\Delta t$ is the time step, $f_{i}^{e q}$ is the equilibrium distribution function, $\tau$ is the non-dimensional relaxation time, and $F_{i}$ represents the force term exerted on the distribution function.

Here D2Q9 and D3Q19 models are respectively employed for two-dimensional and three-dimensional simulations (See Fig. 2), and the discrete velocities $\mathbf{e}_{i}$ are respectively given as $[48,50,52]$

$$
\begin{aligned}
& \mathbf{e}_{i}= \begin{cases}(0,0) & i=0, \\
\left(\cos \frac{\pi(i-1)}{2}, \sin \frac{\pi(i-1)}{2}\right) \frac{\Delta x}{\Delta t} & \text { for } i=1 \text { to } 4, \\
\left(\cos \frac{\pi(i-9 / 2)}{2}, \sin \frac{\pi(i-9 / 2)}{2}\right) \frac{\sqrt{2} \Delta x}{\Delta t} & \text { for } i=5 \text { to } 8,\end{cases} \\
& {\left[\begin{array}{ccccccccccccccccccc}
0 & 1 & -1 & 0 & 0 & 0 & 0 & 1 & -1 & 1 & -1 & 1 & -1 & 1 & -1 & 0 & 0 & 0 & 0 \\
0 & 0 & 0 & 1 & -1 & 0 & 0 & 1 & 1 & -1 & -1 & 0 & 0 & 0 & 0 & 1 & -1 & 1 & -1 \\
0 & 0 & 0 & 0 & 0 & 1 & -1 & 0 & 0 & 0 & 0 & 1 & 1 & -1 & -1 & 1 & 1 & -1 & -1
\end{array}\right] .}
\end{aligned}
$$

The equilibrium distribution function $f_{i}^{e q}$ and the force term $F_{i}$ in Eq. (22) are calculated by $[48,50,52]$

$$
f_{i}^{e q}=w_{i} \rho\left(1+\frac{\mathbf{e}_{i} \cdot \mathbf{u}}{c_{s}^{2}}+\frac{\mathbf{u u}:\left(\mathbf{e}_{i} \mathbf{e}_{i}-c_{s}^{2} \mathbf{I}\right)}{2 c_{s}^{4}}\right),
$$




$$
F_{i}=\left(1-\frac{1}{2 \tau}\right) w_{i}\left(\frac{\mathbf{e}_{i}-\mathbf{u}}{c_{s}^{2}}+\frac{\mathbf{e}_{i} \cdot \mathbf{u}}{c_{s}^{4}} \mathbf{e}_{i}\right) \cdot \mathbf{f}
$$

where $w_{i}$ are the weight factors, $c_{s}=1 / \sqrt{3}$ is the speed of sound, and $\mathbf{f}$ is the body force acting on the fluid including the viscoelastic force and the immersed-boundary introduced force. For the D2Q9 model, $w_{i}$ are given by $w_{0}=4 / 9, w_{i}=1 / 9$ for $i=1-4$, and $w_{i}=1 / 36$ for $i=5-8$; and for the D3Q19 model, $w_{i}$ are given by $w_{0}=1 / 3, w_{i}=1 / 18$ for $i=1-6$, and $w_{i}=1 / 36$ for $i=7-18$.

The relaxation time $\tau$ is related to the solvent dynamic viscosity in Navier-Stokes equations by $[48,50,52]$

$$
\mu_{s} / \rho=\left(\tau-\frac{1}{2}\right) c_{s}^{2} \Delta t
$$

Once the density distribution function is known, the fluid density, velocity and pressure can be obtained by $[48,50,52]$

$$
\begin{gathered}
\rho=\sum_{i} f_{i}, \\
\mathbf{u}=\frac{\sum_{i} \mathbf{e}_{i} f_{i}+0.5 \mathbf{f} \Delta t}{\rho} \\
p=\rho c_{s}^{2}
\end{gathered}
$$

\subsubsection{The lattice Boltzmann method for the constitutive equations of the viscoelastic models}

The approach proposed by Malaspinas et al. [67] is used to solve the constitutive equations of the viscoelastic models. The same type of approach has also been utilized to simulate the flow of liquid crystals by Denniston et al. [76] and Marenduzzo et al. [77, 78]. This approach is based on the 'advection-diffusion' scheme where each component of the conformation tensor $C_{\alpha \beta}$ is calculated by its own distribution functions $h_{\alpha \beta}[67]$.

The single relaxation time lattice Boltzmann method is utilized to solve the constitutive equations [67]

$h_{i \alpha \beta}\left(\mathbf{x}+\boldsymbol{\xi}_{i} \Delta t, t+\Delta t\right)-h_{i \alpha \beta}(\mathbf{x}, t)=-\frac{1}{\varphi}\left[h_{i \alpha \beta}(\mathbf{x}, t)-h_{i \alpha \beta}^{e q}\left(C_{\alpha \beta}, \mathbf{u}\right)\right]+\left(1-\frac{1}{2 \varphi}\right) \frac{G_{\alpha \beta}}{C_{\alpha \beta}} h_{i \alpha \beta}^{e q}\left(C_{\alpha \beta}, \mathbf{u}\right) \Delta t$,

where $\xi_{i}$ is the discrete velocity along the $i$ th direction, $\varphi$ is the relaxation time, $h_{i \alpha \beta}^{e q}$ is the equilibrium distribution function, and $G_{\alpha \beta}$ is the source term which is determined by the constitutive equations.

The equilibrium distribution function $h_{i \alpha \beta}^{e q}$ is calculated by [67]

$$
h_{i \alpha \beta}^{e q}=w_{v i} C_{\alpha \beta}\left(1+\frac{\boldsymbol{\xi}_{i} \cdot \mathbf{u}}{c_{l}^{2}}\right),
$$

where $w_{v i}$ are the weight factors of the lattice, and $c_{l}$ is a scaling factor. $c_{l}$ equals $1 / \sqrt{3}$ and $1 / 2$ in $2 \mathrm{D}$ and $3 \mathrm{D}$ simulations, respectively [67].

Here D2Q5 and D3Q7 models are respectively employed for two-dimensional and three-dimensional simulations [67]. As reported by Malaspinas et al. [67], D2Q5 and D3Q7 models are able to provide accurate solutions of viscoelastic fluid flows. The weight factors $w_{v i}$ and the lattice velocity $\boldsymbol{\xi}_{i}$ are shown in Table 1 and 2 for D2Q5 and D3Q7 models. 
Table 1: Weight factors $w_{v i}$ and lattice velocity $\boldsymbol{\xi}_{i}$ in D2Q5 model

\begin{tabular}{cccccc}
\hline & $i=0$ & $i=1$ & $i=2$ & $i=3$ & $i=4$ \\
\hline$w_{v i}$ & $1 / 3$ & $1 / 6$ & $1 / 6$ & $1 / 6$ & $1 / 6$ \\
$\boldsymbol{\xi}_{i}$ & $(0,0)$ & $(1,0)$ & $(-1,0)$ & $(0,1)$ & $(0,-1)$ \\
\hline
\end{tabular}

Table 2: Weight factors $w_{v i}$ and lattice velocity $\boldsymbol{\xi}_{i}$ in D3Q7 model

\begin{tabular}{cccccccc}
\hline & $i=0$ & $i=1$ & $i=2$ & $i=3$ & $i=4$ & $i=5$ & $i=6$ \\
\hline$w_{v i}$ & $1 / 4$ & $1 / 8$ & $1 / 8$ & $1 / 8$ & $1 / 8$ & $1 / 8$ & $1 / 8$ \\
$\boldsymbol{\xi}_{i}$ & $(0,0,0)$ & $(1,0,0)$ & $(-1,0,0)$ & $(0,1,0)$ & $(0,-1,0)$ & $(0,0,1)$ & $(0,0,-1)$ \\
\hline
\end{tabular}

The conformation tensor $C_{\alpha \beta}$ is calculated from the distribution function $h_{i \alpha \beta}$ [67]

$$
C_{\alpha \beta}=\sum_{i} h_{i \alpha \beta}+\frac{G_{\alpha \beta}}{2} \Delta t
$$

The source term $G_{\alpha \beta}$ is determined according to the constitutive equations employed. For the Oldroyd-B, FENE-CR, and Giesekus models, the source terms are respectively computed by $[21,24,67]$

$$
\begin{gathered}
\boldsymbol{G}=-\frac{1}{\lambda}(\boldsymbol{C}-\boldsymbol{I})+\boldsymbol{C} \cdot \nabla \boldsymbol{u}+(\nabla \boldsymbol{u})^{T} \cdot \boldsymbol{C} \quad(\text { Oldroyd }-\mathrm{B}), \\
\boldsymbol{G}=-\frac{1}{\lambda}(\boldsymbol{C}-\boldsymbol{I})-\frac{\alpha}{\lambda}(\boldsymbol{C}-\boldsymbol{I}) \cdot(\boldsymbol{C}-\boldsymbol{I})+\boldsymbol{C} \cdot \nabla \boldsymbol{u}+(\nabla \boldsymbol{u})^{T} \cdot \boldsymbol{C} \quad \text { (Giesekus), } \\
\boldsymbol{G}=-\frac{F(\boldsymbol{C})}{\lambda}(\boldsymbol{C}-\boldsymbol{I})+\boldsymbol{C} \cdot \nabla \boldsymbol{u}+(\nabla \boldsymbol{u})^{T} \cdot \boldsymbol{C} \quad(\text { FENE }-\mathrm{CR}) .
\end{gathered}
$$

Carrying out the Chapman-Enskog expansion on the lattice Boltzmann scheme for the constitutive equations, the following equation can be recovered [67]

$$
\frac{D C_{\alpha \beta}}{D t}=G_{\alpha \beta}+\kappa \nabla^{2} C_{\alpha \beta}+\frac{\kappa}{c_{l}^{2}} \nabla \cdot\left(C_{\alpha \beta} \partial_{t} \boldsymbol{u}-\boldsymbol{u} \nabla \cdot\left(C_{\alpha \beta} \boldsymbol{u}\right)\right),
$$

where $\kappa$ is a diffusive constant given by $\kappa=c_{l}^{2}(\varphi-0.5) \Delta t$. It is added here to control the growth of the gradient of the conformation tensor and enhance the stability of the simulations [79]. It is a key issue to adopt a suitable value of $\kappa$ in the simulations in this scheme. $\kappa$ should be very small in practice [67, 24]. As suggested by Malaspinas et al. [67], the value of the ratio $(\rho \kappa) / \mu_{p}$ should be in the order of $10^{-6}$, and Lee and Ahn [24] suggested that the ratio $(\rho \kappa) / \mu_{p} \sim 10^{-5}$ can also provide convincing results. In this work, the values of non-dimensional diffusion parameter $\operatorname{Pr}=\frac{\kappa}{\dot{\gamma} L^{2}}$ used in the simulations are in the order of $10^{-5}$ to $10^{-2}$, where $\dot{\gamma}$ is the shear rate and $L$ is the characteristic length. In order to characterise the damping effects, the product $\operatorname{Pr} W i$ is used to describe the ratio of the damping to the relaxation effects. Validations show that $\operatorname{Pr} W i$ should be in the order of $\mathrm{O}\left(10^{-3}\right)$ or less to minimize the damping effects (See details in Section 4.2), allowing simulations involving high $W i$ without any preconditioner. 


\subsection{Numerical methods for the solid structures}

In this work, we use different methods for different types of structural dynamics, to maximize the simplicity, efficiency and effectiveness. Specifically, the finite difference method is used to discretize temporal derivatives. In addition, the finite difference method is used to solve $2 \mathrm{D}$ capsule and 3D flags which can be described by using Cartesian Lagrangian systems and descritized on structured meshes. Finally, the 3D capsule is solved by using the finite element method as it is not convenient to use structured mesh in this case.

\subsubsection{The finite difference method for the 2D capsule membrane model}

The 2D capsule membrane is discretised into $N_{f}$ initially equally spaced nodal points, and the elastic and bending forces exerted on these nodal points are computed by the finite difference method.

The elastic force at $k$ th point is computed as

$$
\left.\frac{d}{d s}(\tau \mathbf{t})\right|_{k}=\frac{\tau_{k+\frac{1}{2}} \mathbf{t}_{k+\frac{1}{2}}-\tau_{k-\frac{1}{2}} \mathbf{t}_{k-\frac{1}{2}}}{\Delta s_{k-\frac{1}{2}, k+\frac{1}{2}}},
$$

where $\Delta s_{k-\frac{1}{2}, k+\frac{1}{2}}$ is the arc length between the $\left(k-\frac{1}{2}\right)$ th point and the $\left(k+\frac{1}{2}\right)$ th point, and it is approximated by the distance between the two points. The $\left(k-\frac{1}{2}\right)$ th point and the $\left(k+\frac{1}{2}\right)$ th point are chosen to be the midpoints of the $(k-1)$ th and $k$ th points and $k$ th and $(k+1)$ th points, respectively.

The bending force at the $k$ th point is computed by

$$
\left.\frac{d}{d s}(q \mathbf{n})\right|_{k}=\frac{q_{k+\frac{1}{2}} \mathbf{n}_{k+\frac{1}{2}}-q_{k-\frac{1}{2}} \mathbf{n}_{k-\frac{1}{2}}}{\Delta s_{k-\frac{1}{2}, k+\frac{1}{2}}}
$$

where $q$ at the $\left(k+\frac{1}{2}\right)$ th point is calculated as

$$
q_{k+\frac{1}{2}}=\frac{M_{k+1}-M_{k}}{\Delta s_{k, k+1}}=\frac{k_{b}[\epsilon(k+1)-\epsilon(k)]}{\Delta s_{k, k+1}},
$$

and similarly, $q$ at the $\left(k-\frac{1}{2}\right)$ th point is given by $q_{k-\frac{1}{2}}=\frac{k_{b}[\epsilon(k)-\epsilon(k-1)]}{\Delta s_{k-1, k}}$.

It should be noted that the arc length used in the equations above cannot be approximated by the initial arc length $\left(s_{0}\right)$ as in Ref. [52], since the capsule may experience large deformation rather than a low stretch ratio.

\subsubsection{The finite element method for the $3 D$ capsule}

The finite element method is used for the 3D capsules. This method is based on triangle elements which are used to discretise the surface of 3D solid particles (including 3D deforming capsules and rigid particles). The discretisation of the surface of the particle starts from a regular icosahedron with 20 equilateral triangles and 12 nodes (see Fig. 3(a)). The mesh is refined by dividing each element into 4 triangle elements. The new nodes on the surface are then projected onto the surface of the solid particle (see Fig. 3(b)). The refinement process is repeated until the Lagrangian mesh spacing is approximately half of that of the Eulerian mesh [47, 80]. A similar method has been used by Ramanujan and Pozrikidis [81], Sui et al. [50] and Krüger et al. [82]. In this study, the spherical solid particle normally has 2562 nodes and 5120 elements (as shown in Fig. 3(c)) which is sufficient to describe the behaviour of the particle [83, 82, 84]. 
(a)

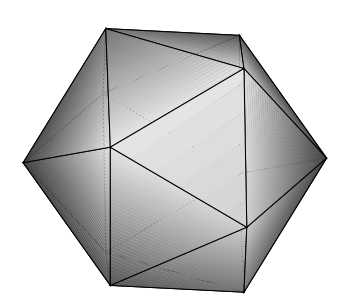

(b)

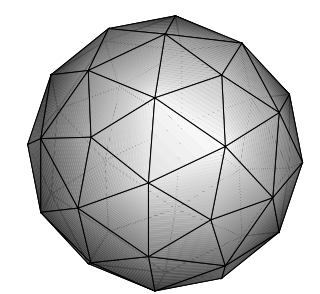

(c)

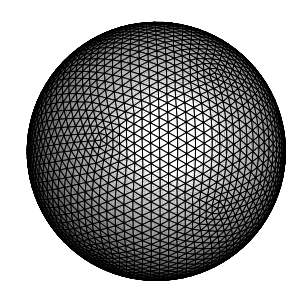

Figure 3: Generating triangle elements on the surface of a sphere: (a) a regular icosahedron, (b) single refinement of each face, (c) resulting sphere with 2562 nodes and 5120 elements.

The internal stretching forces exerted on the nodes of the 3D capsule membrane are computed by a finite element model developed by Charrier et al. [85] and Shrivastava and Tang [86]. Here a brief overview of the model is presented. In this model, the stretching force acting on each node of an element is calculated via the displacement of the deformed element with respect to its prior undeformed state. The deformed and undeformed surface elements are transformed to a common plane, so that the relative displacement of the nodes and the corresponding forces are easily determined (see Fig. 4). It is assumed that the relative displacement $\boldsymbol{d}=\left\{d_{x e}, d_{y e}\right\}$ varies linearly in the plane of the element. $\boldsymbol{d}$ is then computed by

$$
\boldsymbol{d}=N_{i} \boldsymbol{d}_{i}+N_{j} \boldsymbol{d}_{j}+N_{k} \boldsymbol{d}_{k}
$$

where $N_{i}, N_{j}$, and $N_{k}$ are the linear shape functions, and $\boldsymbol{d}_{i}, \boldsymbol{d}_{j}, \boldsymbol{d}_{k}$ are respectively the displacement at the three nodes of the element. $N_{i}$ is calculated as

$$
N_{i}=\frac{a_{i}+b_{i} x_{e}+c_{i} y_{e}}{2 A}
$$

and the coefficients $a_{i}, b_{i}, c_{i}$ and $A$ are given by

$$
a_{i}=x_{e j} y_{e k}-x_{e k} y_{e j}, \quad b_{i}=y_{e j}-y_{e k}, \quad c_{i}=-x_{e j}+x_{e k}, \quad 2 A=a_{i}+b_{i} x_{e i}+c_{i} y_{e i} .
$$

The shape functions $N_{j}$ and $N_{k}$ are obtained by a cyclic change of the subscript in Eq. (42) and (43). Once the shape functions are obtained, the displacement gradients of the elements (e.g., $\partial \boldsymbol{d} / \partial x, \partial \boldsymbol{d} / \partial y)$ can be calculated by differentiating Eq. (41).

The principal extension ratios $\lambda_{1}$ and $\lambda_{2}$ are then calculated according to

$$
\begin{aligned}
& \lambda_{1}^{2}=\frac{1}{2}\left[G_{11}+G_{22}+\sqrt{\left(G_{11}-G_{22}\right)^{2}+4 G_{12}^{2}}\right], \\
& \lambda_{2}^{2}=\frac{1}{2}\left[G_{11}-G_{22}+\sqrt{\left(G_{11}-G_{22}\right)^{2}+4 G_{12}^{2}}\right],
\end{aligned}
$$

where $G_{i j}$ are obtained according to

$$
G_{11}=\left(1+\frac{\partial d_{x e}}{\partial x_{e}}\right)^{2}+\left(\frac{\partial d_{y e}}{\partial x_{e}}\right)^{2}
$$


(a)

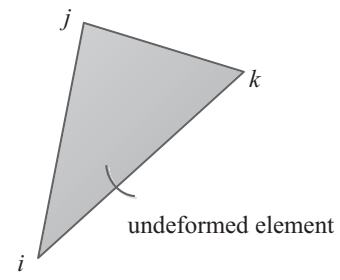

(b)

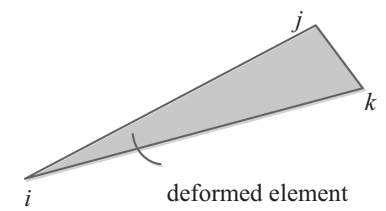

(c)

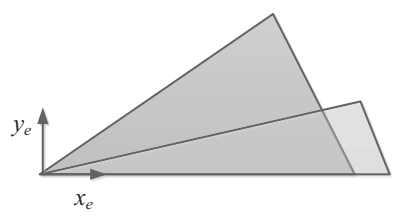

Figure 4: Illustration of the deformation of a triangular element in space, and the comparison of the deformed and the undeformed element in a common plane.

$$
\begin{gathered}
G_{22}=\left(\frac{\partial d_{x e}}{\partial y_{e}}\right)^{2}+\left(1+\frac{\partial d_{y e}}{\partial y_{e}}\right)^{2} \\
G_{12}=G_{21}=\left(1+\frac{\partial d_{x e}}{\partial x_{e}}\right)\left(\frac{\partial d_{x e}}{\partial y_{e}}\right)+\left(1+\frac{\partial d_{y e}}{\partial y_{e}}\right)\left(\frac{\partial d_{y e}}{\partial x_{e}}\right) .
\end{gathered}
$$

The corresponding in-plane elastic force in each element is obtained by applying the principle of virtual work as follows,

$$
f_{e}=-\frac{\delta W^{s}}{\delta \boldsymbol{d}} .
$$

As $f_{e}$ is calculated in the local system, it needs to be transformed to the global coordinates. The force exerted on the fluid by the stretching component can be found as the resultant of the in-plane stretching force $\boldsymbol{f}_{e}$.

The membrane bending force obtained from the bending energy function is computed by [74, $83,87]$

$$
\boldsymbol{f}_{b}=-E_{b}\left[\left(2 H+c_{0}\right)\left(2 H^{2}-2 \kappa_{g}-c_{0} H\right)+2 \Delta_{L B} H\right] \boldsymbol{n},
$$

where $\kappa_{g}$ is the Gaussian curvature, $\boldsymbol{n}$ is the outwards unit normal vector, and $\Delta_{L B}$ is the LaplaceBeltrami operator.

To determine the mean curvature $H$, the Gaussian curvature $\kappa_{g}$ and the Laplace-Beltrami operator $\Delta_{L B}$, we adopt the method proposed by Meyer et al. [88]. In this method, the mean curvature $H$ at the point $\boldsymbol{X}_{i}$ is computed by

$$
H\left(\boldsymbol{X}_{i}\right) \boldsymbol{n}=\frac{1}{4 \sum_{j \in N_{1}(i)} A_{V, j}} \sum_{j \in N_{1}(i)}\left(\cot \alpha_{j}+\cot \gamma_{j}\right)\left(\boldsymbol{X}_{j}-\boldsymbol{X}_{i}\right),
$$

where $\boldsymbol{n}$ is the outer unit normal vector at point $\boldsymbol{X}_{i}, \alpha_{j}$ and $\gamma_{j}$ denote the angles opposite to the edge $\boldsymbol{X}_{i} \boldsymbol{X}_{j}$ (see Fig. 5(a)), $N_{1}(i)$ is the index set of the 1-ring neighbors of point $\boldsymbol{X}_{i}$, and $\sum_{j \in N_{1}(i)} A_{V, j}$ is the summation of the Voronoi region area in each neighbouring triangle of the point $\boldsymbol{X}_{i}$. Here the Voronoi region is defined by the point $\boldsymbol{X}_{i}$, the midpoints of the triangle edges, and the circumcenter of the triangle (see Fig. 5(a)). The area of the Voronoi region for each triangle around point $\boldsymbol{X}_{i}$ (e.g., the one in triangle $\Delta \boldsymbol{X}_{i} \boldsymbol{X}_{j} \boldsymbol{X}_{j+1}$ ) is calculated by 
(a)

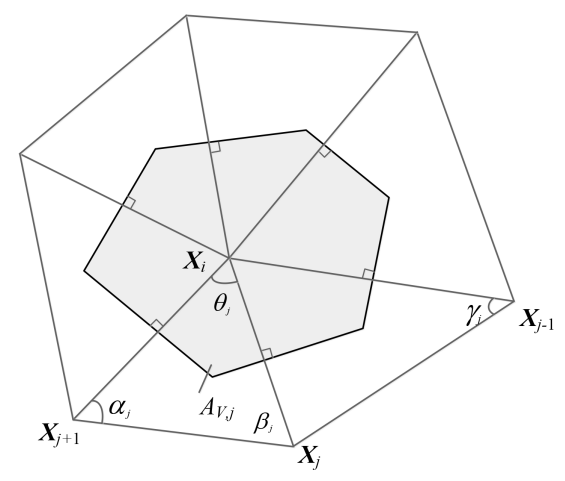

(b)

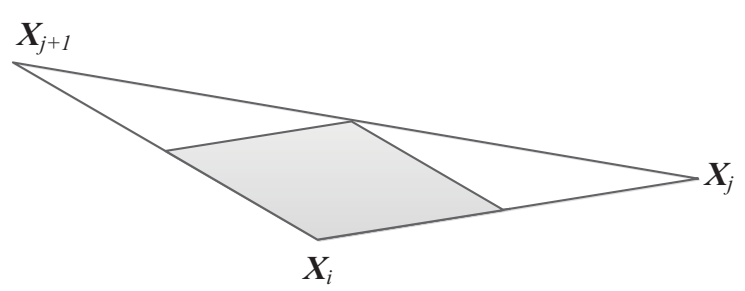

Figure 5: (a) The Voronoi region (shaded) in a 1-ring neighborhood of the vertex. (b) The partitioning region (shaded) of point $\boldsymbol{X}_{i}$ in an obtuse triangle.

$$
A_{V, j}=\frac{1}{8}\left(\cot \alpha_{j}\left\|\boldsymbol{X}_{i}-\boldsymbol{X}_{j}\right\|^{2}+\cot \beta_{j}\left\|\boldsymbol{X}_{i}-\boldsymbol{X}_{j+1}\right\|^{2}\right) .
$$

The Gaussian curvature $\kappa_{g}$ at the point $\boldsymbol{X}_{i}$ is computed as follows,

$$
\kappa_{g}=\frac{1}{\sum_{j \in N_{1}(i)} A_{V, j}}\left(2 \pi-\sum_{j \in N_{1}(i)} \theta_{j}\right),
$$

where $\theta_{j}$ is the angle of the $j^{\text {th }}$ triangle element at the vertex $\boldsymbol{X}_{i}$, as shown in Fig. 5(a).

The Laplace-Beltrami operator of the mean curvature $\Delta_{L B} H$ is estimated by

$$
\Delta_{L B} H=\frac{1}{\sum_{j \in N_{1}(i)} A_{V, j}} \sum_{j \in N_{1}(i)}\left(\cot \alpha_{j}+\cot \gamma_{j}\right)\left(H_{i}-H_{j}\right) .
$$

The Voronoi partitioning scheme is not suitable for triangles with an obtuse angle because the circumcenter of the obtuse triangle does not lie in the internal region of the triangle. For this situation, Meyer et al. [88] used a third vertex which is the midpoint of the edge opposite to the obtuse angle to conduct the partitioning of the triangle (see Fig. 5(b)). A new surface area for $\boldsymbol{X}_{i}$ can now be defined as follows,

$$
A_{m i x}=\sum_{j \in N_{1}(i)} F A\left(T_{j}\right)
$$

where

$$
F A\left(T_{j}\right)=\left\{\begin{array}{lr}
A_{V, j} & T_{j} \text { is acute } \\
\operatorname{Area}\left(T_{j}\right) / 2 & T_{j} \text { is obtuse and the angle of } T_{j} \text { at } X_{i} \text { is obtuse } \\
\operatorname{Area}\left(T_{j}\right) / 4 & T_{j} \text { is obtuse and the angle of } T_{j} \text { at } X_{i} \text { is acute }
\end{array}\right.
$$

$T_{j}$ denotes the $j^{\text {th }}$ triangle at the vertex $\boldsymbol{X}_{i}$, and $\operatorname{Area}\left(T_{j}\right)$ is the area of the triangle $T_{j}$. The summation $\sum_{j \in N_{1}(i)} A_{V, j}$ in Eq. (51), (53), and (54) can be replaced by $A_{m i x}$.

To handle the capsule-capsule and capsule-wall collisions, an artificial repulsion force is introduced as, 


$$
\boldsymbol{f}_{c}(\mathbf{s}, t)=k_{c} \delta_{h}\left(\mathbf{X}(\mathbf{s}, t)-\mathbf{X}^{\prime}\left(\mathbf{s}^{\prime}, t\right)\right) \frac{\mathbf{X}-\mathbf{X}^{\prime}}{\left|\mathbf{X}-\mathbf{X}^{\prime}\right|},
$$

where $k_{c}$ is a positive constant, $\mathbf{X}(\mathrm{s}, \mathrm{t})$ and $\mathbf{X}^{\prime}(\mathrm{s}, t)$ are respectively the position vectors of two nodes, and $\delta_{h}\left(\mathbf{X}(\mathbf{s}, t)-\mathbf{X}^{\prime}(\mathbf{s}, t)\right)$ is a smoothed approximation of the Dirac delta function (see details in Section 3.3). The artificial repulsion force utilized in this work is similar in form to that used in Ref. [89].

It should be noted that the finite element method was adopted for 3D capsules due to its more straight forward computation of elastic and bending forces on 3D deforming structures, as well as availability of previous studies $[50,83,81]$ for validation.

\subsubsection{The finite difference method for rigid particle equations}

The Euler scheme is used to solve the rigid particle equations as follows,

$$
\begin{aligned}
& m \frac{\boldsymbol{V}^{n+1}-\boldsymbol{V}^{n}}{\Delta t}=\boldsymbol{F}_{e x t}^{n}, \\
& {[I] \frac{\boldsymbol{\Omega}^{n+1}-\boldsymbol{\Omega}^{n}}{\Delta t}=\boldsymbol{T}_{e x t}^{n},}
\end{aligned}
$$

where $\Delta t$ is the time step.

\subsubsection{The finite difference method for the 3D flag dynamics}

The flag is treated as several parallel successively connected filaments. This treatment is similar to that in Refs. $[90,66]$. To simplify the calculation, a predicted position $\mathbf{X}^{*}$ is introduced, $\mathbf{X}^{*}=2 \mathbf{X}^{n}-\mathbf{X}^{n-1}$. The stretching force terms $\frac{\partial}{\partial s_{i}}\left(\sigma_{i j} \frac{\partial \mathbf{X}}{\partial s_{j}}\right)$ for $i=j=2 ; i=1, j=2$; and $i=2, j=1$, and the bending force terms $\frac{\partial^{2}}{\partial s_{i} s_{j}}\left(\kappa_{i j}^{B} \frac{\partial^{2} \mathbf{X}}{\partial s_{i} s_{j}}\right)$ for $i=j=2 ; i=1, j=2$; and $i=2, j=1$ are taken as the external force terms of the filaments determined by $\mathbf{X}^{*}$. Then the governing equation for the flexible flag becomes

$$
\rho_{s} \frac{\partial^{2} \mathbf{X}}{\partial t^{2}}=\frac{\partial}{\partial s_{1}}\left(T \frac{\partial \mathbf{X}}{\partial s_{1}}\right)-\frac{\partial^{2}}{\partial s_{1}^{2}}\left(\kappa_{11}^{B} \frac{\partial^{2} \mathbf{X}}{\partial s_{1}^{2}}\right)+\boldsymbol{F}_{H}+\boldsymbol{F}_{e x}\left(\mathbf{X}^{*}\right),
$$

where $T$ is the tension force along the filament axis, and $\boldsymbol{F}_{e x}\left(\mathbf{X}^{*}\right)$ is the summation of the stretching and bending forces mentioned above. These force terms are respectively computed as [91]

$$
\begin{gathered}
\frac{\partial}{\partial s_{2}}\left(\sigma_{22} \frac{\partial \mathbf{X}^{*}}{\partial s_{2}}\right)=D_{2}^{-}\left(\sigma_{22} D_{2}^{+} \mathbf{X}^{*}\right), \\
\frac{\partial}{\partial s_{1}}\left(\sigma_{12} \frac{\partial \mathbf{X}^{*}}{\partial s_{2}}\right)=\frac{1}{2}\left[D_{1}^{-}\left(\sigma_{12}^{+} D_{2}^{+} \mathbf{X}^{*}\right)+D_{1}^{-}\left(\sigma_{12}^{-} D_{2}^{-} \mathbf{X}^{*}\right)\right], \\
\frac{\partial}{\partial s_{2}}\left(\sigma_{21} \frac{\partial \mathbf{X}^{*}}{\partial s_{1}}\right)=\frac{1}{2}\left[D_{2}^{-}\left(\sigma_{21}^{+} D_{1}^{+} \mathbf{X}^{*}\right)+D_{2}^{-}\left(\sigma_{21}^{-} D_{1}^{-} \mathbf{X}^{*}\right)\right], \\
\frac{\partial^{2}}{\partial s_{2}^{2}}\left(\kappa_{22}^{B} \frac{\partial^{2} \mathbf{X}^{*}}{\partial s_{2}^{2}}\right)=D_{22}^{0}\left(\kappa_{22}^{B} D_{22}^{0} \mathbf{X}^{*}\right), \\
\frac{\partial^{2}}{\partial s_{1} s_{2}}\left(\kappa_{12}^{B} \frac{\partial^{2} \mathbf{X}^{*}}{\partial s_{1} s_{2}}\right)=\frac{\partial^{2}}{\partial s_{2} s_{1}}\left(\kappa_{21}^{B} \frac{\partial^{2} \mathbf{X}^{*}}{\partial s_{2} s_{1}}\right)=D_{12}^{-}\left(\kappa_{12}^{B} D_{12}^{+} \mathbf{X}^{*}\right),
\end{gathered}
$$


where $\left[D_{1}^{-} \phi\right]_{i, j},\left[D_{1}^{+} \phi\right]_{i, j},\left[D_{22}^{0} \phi\right]_{i, j},\left[D_{12}^{+} \phi\right]_{i, j}$, and $\left[D_{12}^{-} \phi\right]_{i, j}$ are repsectively defined as

$$
\begin{gathered}
{\left[D_{1}^{+} \phi\right]_{i, j}=\left(\phi_{i+1, j}-\phi_{i, j}\right) / \Delta s_{1},} \\
{\left[D_{1}^{-} \phi\right]_{i, j}=\left(\phi_{i, j}-\phi_{i-1, j}\right) / \Delta s_{1},} \\
{\left[D_{22}^{0} \phi\right]_{i, j}=\left(\phi_{i, j+1}-2 \phi_{i, j}+\phi_{i, j-1}\right) / \Delta s_{1}^{2},} \\
{\left[D_{12}^{+} \phi\right]_{i, j}=\left(\phi_{i+1, j+1}-\phi_{i+1, j}-\phi_{i, j+1}+\phi_{i, j}\right) /\left(\Delta s_{1} \Delta s_{2}\right),} \\
{\left[D_{12}^{-} \phi\right]_{i, j}=\left(\phi_{i, j}-\phi_{i, j-1}-\phi_{i-1, j}+\phi_{i-1, j-1}\right) /\left(\Delta s_{1} \Delta s_{2}\right) .}
\end{gathered}
$$

The filaments are assumed to be inextensible along the $s_{1}$ direction, and the Poisson equation for the tension force $T$ is [91]

$$
\frac{\partial \mathbf{X}}{\partial s_{1}} \cdot \frac{\partial^{2}}{\partial s_{1}^{2}}\left(T \frac{\partial \mathbf{X}}{\partial s_{1}}\right)=\frac{1}{2} \frac{\partial^{2}}{\partial t^{2}}\left(\frac{\partial \mathbf{X}}{\partial s_{1}} \cdot \frac{\partial \mathbf{X}}{\partial s_{1}}\right)-\rho_{s} \frac{\partial^{2} \mathbf{X}}{\partial t \partial s_{1}} \cdot \frac{\partial^{2} \mathbf{X}}{\partial t \partial s_{1}}-\frac{\partial \mathbf{X}}{\partial s_{1}} \cdot \frac{\partial}{\partial s_{1}}\left[\frac{\partial^{2}}{\partial s_{1}^{2}}\left(\kappa_{11}^{B} \frac{\partial^{2} \mathbf{X}}{\partial s_{1}^{2}}\right)+\boldsymbol{F}_{H}+\boldsymbol{F}_{e x}\right] .
$$

The implicit second-order finite-difference scheme is used to discretise the Eq. (60) in time as used in Ref. [89]

$$
\frac{\mathbf{X}^{n+1}-2 \mathbf{X}^{n}+\mathbf{X}^{n-1}}{\Delta t^{2}}=\frac{\partial}{\partial s_{1}}\left(T^{n+\frac{1}{2}} \frac{\partial \mathbf{X}^{n+1}}{\partial s_{1}}\right)-\frac{\partial^{2}}{\partial s_{1}^{2}}\left(\kappa_{11}^{B} \frac{\partial^{2} \mathbf{X}^{*}}{\partial s_{1}^{2}}\right)+\boldsymbol{F}_{e x}\left(\mathbf{X}^{*}\right)+\boldsymbol{F}_{H}^{n},
$$

where $\Delta t$ is the time step, and $T^{n+\frac{1}{2}}$ is calculated by solving Eq. (71) (Details can be found in Ref. [89]). The filament position at new time step $\mathbf{X}^{n+1}$ is obtained by solving Eq. (72).

\subsection{The immersed boundary method}

The interaction between the fluid and the deformable structures is achieved by using the immersed boundary method. In this method, a set of discrete marker points are used to represent the boundary geometry $[45,50]$. The effects of the immersed boundaries are taken into account by spreading the Lagrangian force onto the ambient fluid as a body force [45, 50],

$$
\boldsymbol{f}(\mathbf{x}, t)=\int \boldsymbol{F}_{L}(\mathbf{s}, t) \delta_{h}(\mathbf{x}-\mathbf{X}(\mathbf{s}, t)) d \mathbf{s}
$$

where $\boldsymbol{f}(\mathbf{x}, t)$ is the fluid body force density, $\boldsymbol{F}_{L}(\mathbf{s}, t)$ is the lagrangian force density, $\delta_{h}(\mathbf{x}-\mathbf{X}(\mathbf{s}, t))$ is a smoothed approximation of the Dirac delta function. In the two-dimensional study, it is chosen to be

$$
\delta_{h}(\mathbf{x}-\mathbf{X}(\mathbf{s}, t))=\frac{1}{h^{2}} \phi\left(\frac{x-X(\mathbf{s}, t)}{h}\right) \phi\left(\frac{y-Y(\mathbf{s}, t)}{h}\right),
$$

and in the three-dimensional study, the following form is used,

$$
\delta_{h}(\mathbf{x}-\mathbf{X}(\mathbf{s}, t))=\frac{1}{h^{3}} \phi\left(\frac{x-X(\mathbf{s}, t)}{h}\right) \phi\left(\frac{y-Y(\mathbf{s}, t)}{h}\right) \phi\left(\frac{z-Z(\mathbf{s}, t)}{h}\right),
$$


where $h$ is the mesh spacing and

$$
\phi(r)=\left\{\begin{array}{lr}
\frac{1}{8}\left(3-2|r|+\sqrt{1+4|r|-4 r^{2}}\right) & 0 \leq|r| \leq 1, \\
\frac{1}{8}\left(5-2|r|+\sqrt{-7+12|r|-4 r^{2}}\right) & 1 \leq|r| \leq 2, \\
0 & |r|>2 .
\end{array}\right.
$$

For structures with mass (e.g., flag flapping and rigid particle flows), the Lagrangian force $\boldsymbol{F}_{L}$ is determined by

$$
\boldsymbol{F}_{L}=\alpha \frac{\boldsymbol{U}-\boldsymbol{u}_{i b}}{\Delta t}
$$

where $\boldsymbol{U}$ is the structure velocity obtained by solving the structure dynamics, $\Delta t$ is the time step, $\alpha$ is a positive constant, and $\boldsymbol{u}_{i b}$ is the interpolated velocity flow field,

$$
\mathbf{u}_{i b}(\mathbf{s}, t)=\int \mathbf{u}(\mathbf{x}, t) \delta_{h}(\mathbf{x}-\mathbf{X}(\mathbf{s}, t)) d \mathbf{x} .
$$

For massless structures (e.g., capsules), the position of the solid structure is first updated by $\frac{\partial \mathbf{X}(\mathbf{s}, t)}{\partial t}=\mathbf{u}_{i b}(\mathbf{s}, t)$, and then $\boldsymbol{F}_{L}$ is determined by the elastic forces.

\section{Validation}

Several validation cases including a 2D rigid particle migration in a Giesekus Couette flow, a 3D rigid particle rotation in an Oldroyd-B shear flow, a 3D rigid particle settling in a FENE-CR fluid, 2D and 3D capsules deformation in a Newtonian shear flow, and a 3D flag flapping in a Newtonian free stream are conducted to validate the current method.

\subsection{A 2D neutrally buoyant particle migration in a Giesekus Couette flow}

The dynamics of a 2D neutrally buoyant particle migration in a Giesekus Couette flow is considered here. The definition of the physical problem and the associated coordinate system is shown in Fig. 6(a). The radius of the circular particle is $R$, and the computational domain is $[-10 R, 10 R] \times[0,20 R]$. Periodic boundary conditions are applied at the inlet and outlet. A constant velocity $U_{c}$ is imposed at the bottom boundary, while $-U_{c}$ is applied at the top boundary. The grid spacing $h=\Delta x=\Delta y=0.05 R$ is used. The non-dimensional parameters are the Reynolds number $R e=\rho_{f} \dot{\gamma}(2 R)^{2} / \mu_{0}$, the Weissenberg number $W i=\lambda \dot{\gamma}$, the ratio of the solvent to the total viscosity $\beta=\mu_{s} / \mu_{0}$, the non-dimensional diffusion parameter $\operatorname{Pr}=\kappa / \dot{\gamma}(2 R)^{2}$, and the mobility parameter for the Giesekus fluid $\alpha$, where $\rho_{f}$ is the density of the fluid, $\dot{\gamma}$ is the shear rate, $\lambda$ is the polymer relaxation time, $\kappa$ is the diffusive constant, $\mu_{0} \equiv \mu_{s}+\mu_{p}$ is the zero-shear viscosity of the fluid, and $\mu_{s}$ and $\mu_{p}$ are respectively the viscosities of the solvent and the polymer. Note that $\operatorname{Pr}$ is introduced to measure the effect of the diffusion introduced in Eq. (37).

Fig. 6 shows the time evolution of the angular velocity of the rigid particle at initial position $y_{0}=0.2 W$ for $R e=0.011, W i=1.0, \beta=0.1$, and $\alpha=0.2$. To determine a suitable value of the diffusive constant $\kappa$ in Eq. (37), two different non-dimensional values of $P r$ are used in the simulations: $6.25 \times 10^{-4}$ and $6.25 \times 10^{-5}$. As shown in Fig. 6(b), the present method successfully predicts the circular particle migration in the Giesekus Couette flow. Even though slight initial discrepancy is observed, the trend and the steady value of the angular velocity in present study agree well with those in previous numerical studies on rigid particles by a 2D LBM-SPM [24] and a 2D ALE-FEM formulation [92]. In addition, the results are independent of $\mathrm{Pr}$. 
(a)

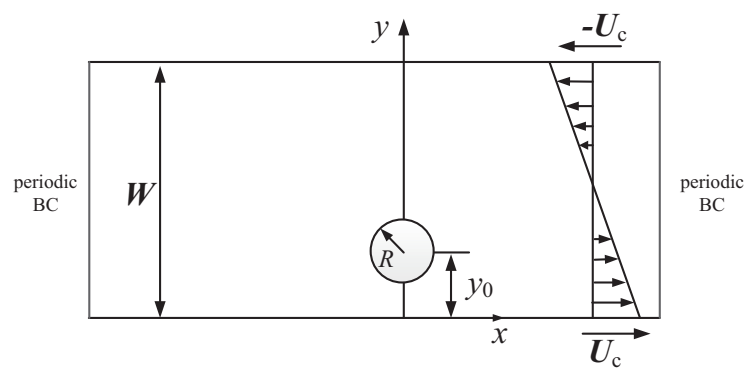

(b)

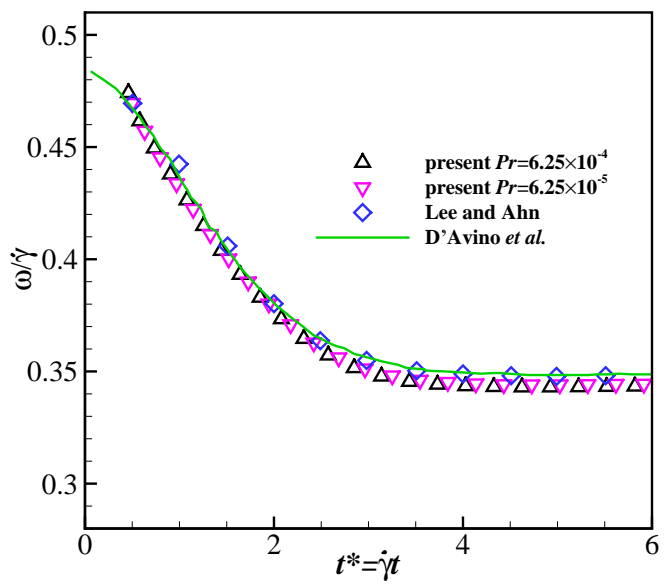

Figure 6: (a) Schematic illustration of a 2D buoyant particle migration in a Giesekus Couette flow. (b) The particle angular velocity as a function of non-dimensional time for starting position $y_{0}=0.2 \mathrm{~W}$ at different values of nondimensional diffusion parameter $\operatorname{Pr}$.

\subsection{A 3D neutrally buoyant spherical particle rotation in an Oldroyd-B shear flow}

To further validate the present method, the rotation of a neutrally buoyant spherical particle in an Oldroyd-B shear flow is considered in this section. As shown in Fig. 7(a), the simulation is conducted in a cuboid domain of $[0,16 R] \times[0,8 R] \times[0,8 R]$ and the center of the particle is initially placed at $(8 R, 4 R, 4 R)$. A simple shear flow is applied to the computational domain by moving the plates at $z=-4 R$ and $z=4 R$ in opposite directions in the $x$-direction but with the same speed $U_{c}$. Periodic boundary conditions are applied at the other four boundaries. The nondimensional parameters are defined as: the Reynolds number $R e=\rho_{f} \dot{\gamma}(2 R)^{2} / \mu_{0}$, the Weissenberg number $W i=\lambda \dot{\gamma}$, the ratio of the solvent to the total viscosity $\beta=\mu_{s} / \mu_{0}$, and the non-dimensional diffusion parameter $\operatorname{Pr}=\kappa / \dot{\gamma}(2 R)^{2}$.

Following the study by Goyal and Derksen [21], the grid spacing in the simulations is $h=\Delta x=$ $\Delta y=\Delta z=R / 6$. Fig. 7(b) shows the steady particle rotation angular velocities at different $W i$ and $\operatorname{Pr}$ for $R e=0.025$ and $\beta=0.5$. It is found that the steady angular velocity of the spherical particle decreases monotonically with increasing $W i$ which means the viscoelasticity of the fluid hinders the rotation of the particle in the Oldroyd-B shear flow. The angular velocities of the particle here show good agreement with previous numerical results by Snijkers et al. [23] using FEM-ALE, Chiu et al. [41] using the distributed Lagrange multiplier/fictitious domain method, and Goyal and Derksen [21] using an the immersed boundary-lattice Boltzmann-finite volume method (IBLBM-FVM) demonstrating the accuracy of the present method to simulate 3D particle motion in viscoelastic fluids. Note that only a rigid particle at $W i$ up to 5 was considered in Refs. [21,23,35], while the current method is able to simulate flexible particle flows at Wi up to 100 which will be discussed in Section 5. It is also found that when $\operatorname{Pr} W i \leq 0.001$, decreasing $\operatorname{Pr} W i$ does not have a significant influence on the angular velocity of the particle. This means the damping effects on the simulations are insignificant compared with the relaxation effects when the product $\operatorname{Pr} W i$ is in the order of $\mathrm{O}\left(10^{-3}\right)$ or less. 
(a)

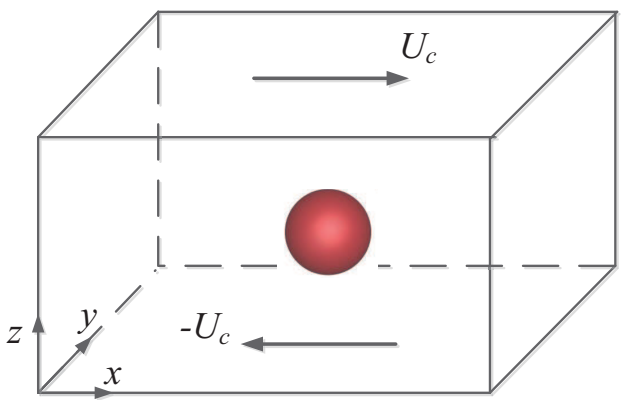

(b)

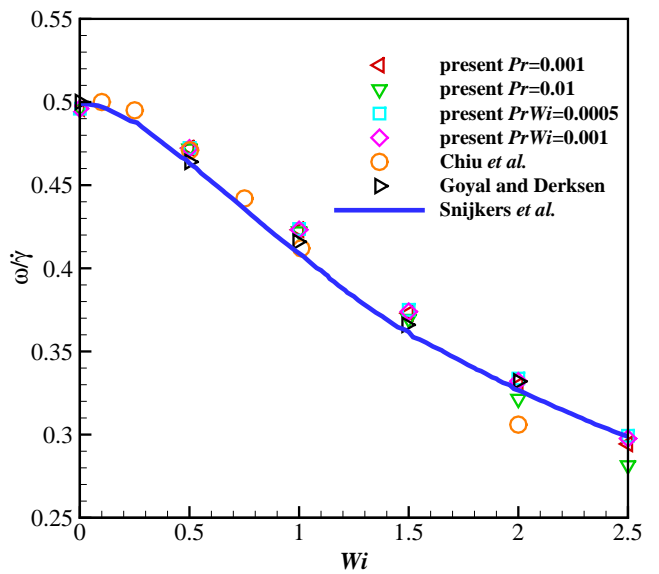

Figure 7: (a) Schematic illustration of a spherical particle rotation in an Oldroyd-B shear flow. (b) The effect of $W i$ on the particle steady rotation angular velocity.

\subsection{A spherical particle settling under gravity in a FENE-CR fluid}

Goyal and Derksen [21] numerically investigated a spherical particle settling in FENE-CR fluids. Following their research, a numerical study of a spherical particle with diameter $d$ sedimentation under gravity in FENE-CR fluids is presented in this section. The physical problem and the coordinate definition of the present simulation is shown in the inset of Fig. 8(a). The computation domain is $[0, L] \times[0, L] \times[0, H](H=4 L)$, and the gravity is applied in the negative $z$ direction. The particle is initially released at the center of the cross-section of the channel and $z=0.8 H$ with zero velocity. The parameters used are based on those used in Goyal and Derksen [21]: the Reynolds number $R e_{\infty}=\frac{\rho_{f} U_{t} d}{\mu_{0}}=0.36$, the Weissenberg number $W i=\lambda \frac{U_{t}}{d}$, the density ratio $\frac{\rho_{p}}{\rho_{f}}=2.5$, the blockage ratio $\frac{d}{L}=0.25$, the ratio of the solvent to the total viscosity $\beta=\frac{\mu_{s}}{\mu_{0}}=0.1$, and the dimensionless extension length $L_{p}^{2}=10$, where $\rho_{p}$ is the density of the particle, and $U_{t}=\frac{\left(\rho_{p}-\rho_{f}\right) g d^{2}}{18 \mu_{0}}$ with $g$ being the gravitational acceleration. The differences are in the height of the computational domain $(H=4 L$ in this study and $H=10 L$ in [21]) and the mesh size $(L=64 \Delta x$ and $128 \Delta x$ in this study and $L=48 \Delta x$ in [21]). The elastic number $\mathrm{El}=W i R e=\frac{\lambda \mu_{0}}{\rho_{f} d^{2}}$ is defined to quantify the elasticity of the fluids. The diffusive constant $\kappa$ employed is $5 \times 10^{-6}$.

The dependence of the particle settling velocity on the spatial resolution and the characteristic velocity is shown in Fig. 8(a) and (b). It is found that the particle settling velocity tends to decreases with the decrease of the characteristic velocity and the increase of the mesh size, and converges at $U_{t}=0.004$ (in LBM units) and $L=128 \Delta x$. In addition, present results agree well with the results from Goyal and Derksen [21] (as shown in Fig. 8(c)). It should be note that larger characteristic velocities are used $\left(U_{t}=0.016\right.$ for $W i=1.0$ and $U_{t}=0.02$ for $\left.W i=2.0\right)$ to match the previous results (in Fig. 8(c)), and it is impossible to conduct further comparison since the configurations (e.g., $U_{t}$ and $P r$ ) are not clear in the reference. The relationship between the dimensionless time of the instant of the maximum velocity $\left(t_{p} / \lambda\right)$ and the elastic number El is exhibited in Fig. 8(d). As indicated by Goyal and Derksen [21], there is a linear relationship between $t_{p} / \lambda$ and $1 / \sqrt{\mathrm{El}}$, and the slope is approximately 0.6. It is found in Fig. 8(c), the present $t_{p} / \lambda$ and $1 / \sqrt{\mathrm{El}}$ show the linear relationship as well, and the slope is approximately 0.668. 
(a)

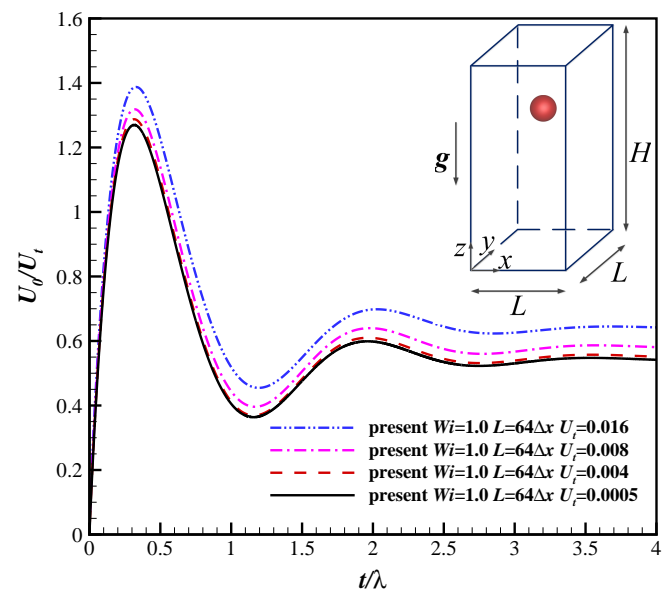

(c)

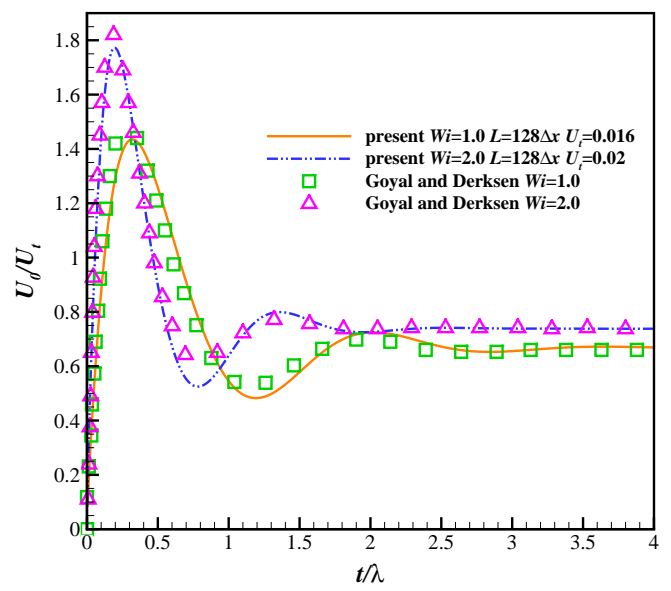

(b)

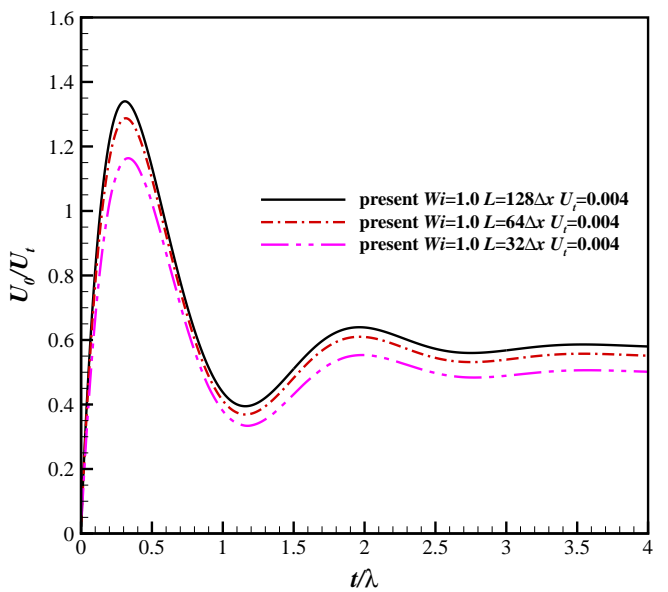

(d)

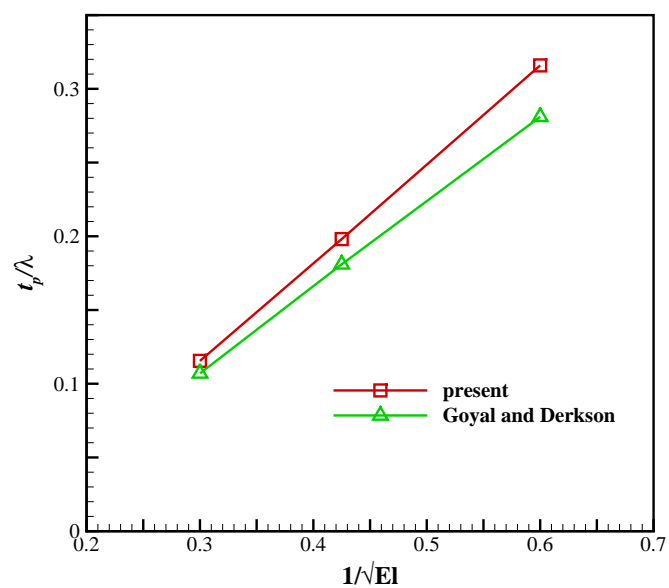

Figure 8: (a) The time evolution of the particle settling velocity in a FENE-CR fluid at different characteristic velocities at $W i=1.0$. Inset: Schematic illustration of a spherical capsule settling in a square channel filled with a FENE-CR fluid. (b) The time evolution of the particle settling velocity in a FENE-CR fluid at different spatial resolutions at $W i=1.0$. (c) The comparison of present results and results from Goyal and Derksen [21] for $W i=1.0$ and 2.0. (d) The relationship between the dimensionless time of the moment of the maximum of the particle settling velocity $\left(t_{p} / \lambda\right)$ and the elasticity number El.

\subsection{A 2D capsule deformation in a Newtonian shear flow}

In this section, the deformation of a 2D circular capsule in a Newtonian shear flow is investigated to validate the present $2 \mathrm{D}$ flexible capsule model. The definition of the flow geometry and the associated coordinate system are shown in Fig. 9. The initial radius of the capsule is $R$, and the computational domain is $[0,16 R] \times[0,16 R]$. The boundary conditions are shown in Fig. 9 . The Reynolds number is defined as $R e=\rho \dot{\gamma}(2 R)^{2} / \mu$. Another two non-dimensional parameters which play important roles on the deformation of the capsule are the non-dimensional shear rate $G=\mu \dot{\gamma} R / k_{s}$, and the non-dimensional bending modulus $e_{b}=k_{b} /\left(R^{2} k_{s}\right)$.

The grid spacing used is $h=\Delta x=\Delta y=0.05 R$. The Reynolds number used is 0.05 , the non-dimensional shear rate is 0.04 , and the non-dimensional bending moduli are $0,0.025,0.05,0.1$ and 0.2. The Taylor deformation parameter $D_{x y}$ and inclination angle $\theta$ are introduced here to 


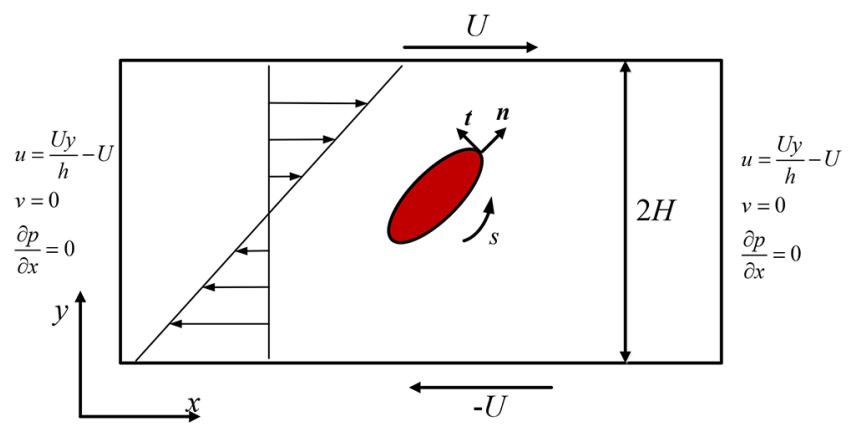

Figure 9: Schematic figure for 2D elastic capsule deformation in a Newtonian shear flow.

(a)

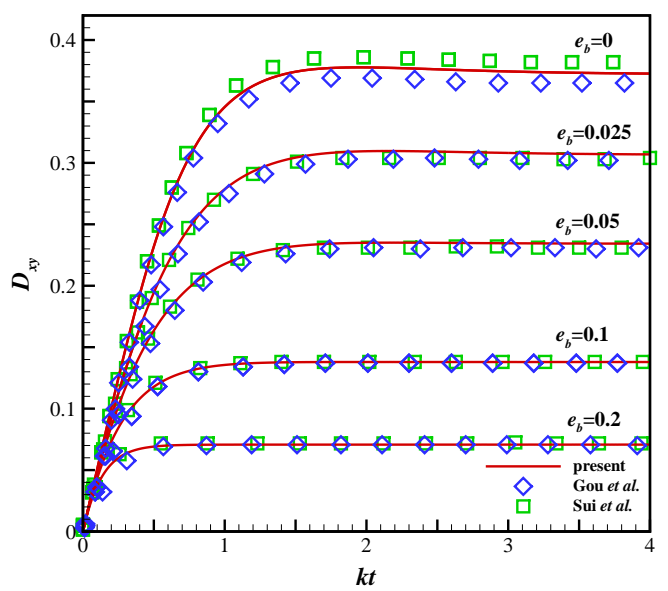

(b)

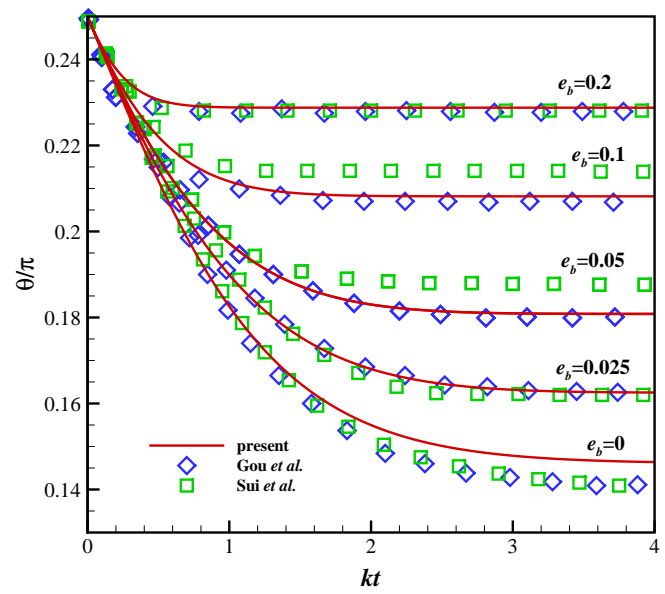

Figure 10: The temporal evolution of (a) Taylor deformation parameter and (b) inclination angle for $G=0.04$.

measure the deformation of the capsule. The Taylor deformation parameter is defined as $D_{x y}=$ $(L-W) /(L+W)$, where $L$ and $W$ are the length and width of the capsule in the shear plane, respectively. The inclination angle $\theta$ (scaled by $\pi$ ) is defined as the angle between the maximum axis of the capsule and the positive $x$-axis.

The temporal evolution of the Taylor deformation parameter and the inclination angle are compared with the results by Sui et al. [68] on 2D IB-LBM and Gou et al. [93] on 2D IB-finite difference method in Fig. 10. It is observed that the present results show very good agreement with previous data in terms of Taylor deformation parameter, while the inclination angle is within the variability between the two previous studies.

\subsection{A $3 D$ non-spherical capsule deformation in a Newtonian shear flow}

The deformation of a 3D oblate spheroidal capsule in a Newtonian shear flow is presented in this section to validate the 3D capsule model in this work. Compared with the study of a spherical capsule, the study of a non-spherical capsule is more demanding since such a capsule can exhibit more complex behaviours due to its anisotropic structure. The surface mesh of the oblate spheroidal capsule is shown in Fig. 11(a). The oblate spheroidal capsule possesses two equivalent 
major axes and a minor axis, and the aspect ratio $b / a$ can be used to characterize the shape of the oblate spheroidal capsule, where $a$ and $b$ represent the length of semi-major and semi-minor axes, respectively. The definition of the flow geometry and the coordinate system are the same as those used in Section 4.2. The computational domain used in this case is $[0,10 R] \times[0,4 R] \times[0,10 R]$, and $R$ is the equivalent radius of a sphere which has the same volume as that of the oblate spheroidal capsule. A simple shear flow is applied to the computational domain by moving the plates at $z=-5 R$ and $z=5 R$ in opposite directions in $x$-direction but with the same speed $U_{c}$. Periodic boundary conditions are applied at the other four boundaries. The non-dimensional parameters in this case are the Reynolds number $R e=\rho(\dot{\gamma} R) R / \mu$ and the non-dimensional shear rate $G=$ $\mu \dot{\gamma} R / E_{s}$. It should be noted that the bending rigidity is neglected here. The validation of the bending model of the 3D capsule is presented in Section 4.6.

The grid spacing used is $h=\Delta x=\Delta y=\Delta z=0.1 R$. The Reynolds number is 0.025 , and the non-dimensional shear rates are 0.1 and 0.2. The initial inclination angle of the capsule (with respect to $x$-axis) is set to be $\pi / 4$. The Taylor deformation parameter $D_{x y}$ and inclination angle $\theta$ are examined here to measure the deformation of the capsule. The Taylor deformation parameter is defined as $D_{x y}=(L-W) /(L+W)$, where $L$ and $W$ are the lengths of semi-minor and semi-major axes of the center plane (i.e., $y=2 R$ ). The inclination angle $\theta$ is defined as the angle between the major semi-axis of the capsule and the positive $x$-axis.

Fig. 11(b) and (c) give the time histories of $D_{x y}$ and $\theta$ for an initially oblate spheroidal capsule of $b / a=0.9$ with a Neo-Hookean membrane. Both $D_{x y}$ and $\theta$ experience oscillation previously observed as a swinging motion [94], in contrast to the usual behaviour of a spherical capsule in a Newtonian shear flow which undergoes steady tank-treading movement. In addition, the present results show good agreement with previous numerical results from Sui et al. [50] using 3D IB-LBM and Ramanujan and Pozrikidis [81] using the boundary element method, as shown in Fig. 11(b) and (c). Note that both references only considered Newtonian fluids.

\subsection{The orientation of a prolate vesicle in a Newtonian shear flow}

In this section, the orientation of a prolate vesicle in a Newtonian shear flow is investigated to further validate the present 3D capsule model. A vesicle is a droplet enclosed by a membrane of a lipid layer, and conserves constant volume $V$ and surface area $S$. When placed in a linear shear flow, the dynamics of the vesicle is controlled by three non-dimensional parameters: the excess area $\Delta=S / R^{2}-4 \pi$, the ratio of the internal to external fluid viscosity $\widehat{\lambda}=\mu_{\text {int }} / \mu_{\text {ext }}$, and the capillary number $\chi=\mu_{\text {ext }} \dot{\gamma} R^{3} / E_{b}$, where $R$ is the radius of the sphere having the same volume to that of the vesicle, and $\mu_{i n t}$ and $\mu_{e x t}$ are respectively the dynamic viscosities of the internal and external fluids of the vesicle. Following the investigation of Yazdani and Bagchi [83], the Skalak's (SK) law with $E_{s}=0$ is employed to calculate the in-plane elastic force.

The computational domain shown schematically in Fig. $7(\mathrm{a})$ is $[0,10 R] \times[0,6 R] \times[0,10 R]$. Periodic boundary conditions are applied in $x$ and $y$ directions. The grid spacing of $h=\Delta x=$ $\Delta y=\Delta z=R / 15$ is used, and the Reynolds number $R e=\rho \dot{\gamma} R^{2} / \mu_{\text {ext }}$ is 0.025 . The excess area $\Delta$ ranges from 0.125 to 2.17 , the viscosity ratio $\hat{\lambda}=1.0$, and the capillary number $\chi=10$. The non-dimensional area dilation modulus $G=\mu_{e x t} \dot{\gamma} R^{2} / E_{a}=0.003$ is used to ensure constant surface area.

The inclination angles $\theta$ (radians scaled by $\pi$ ) of the vesicle with respect to the positive $x$ axis as a function of the excess area $\Delta$ are shown in Fig. 12. The present results are compared with the numerical results from Yazdani and Bagchi [83], Zhao and Shaqfeh [95], and Kraus et 


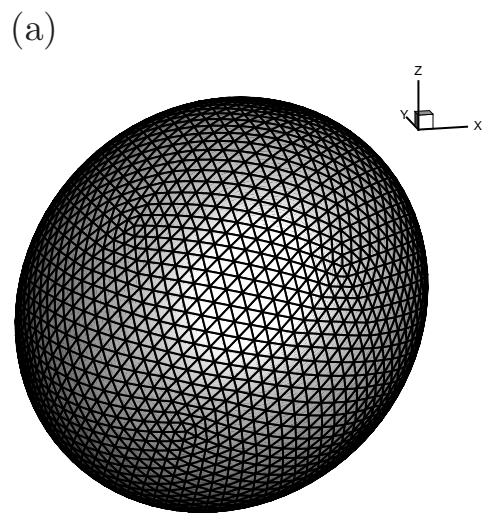

(b)

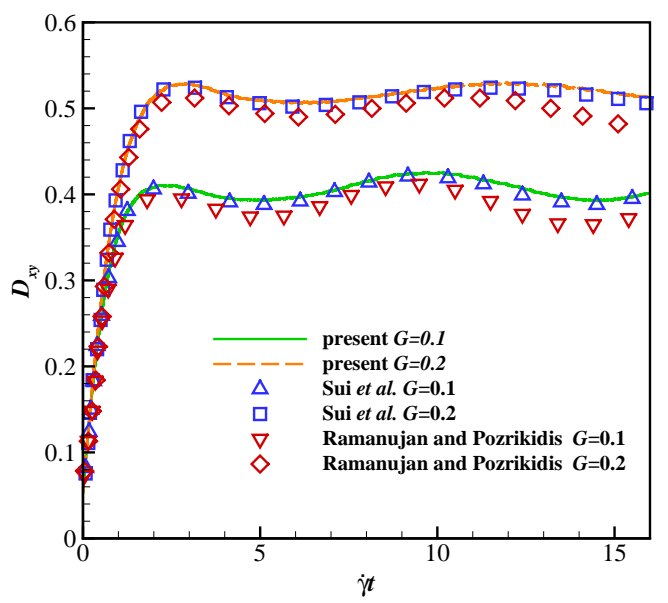

(c)

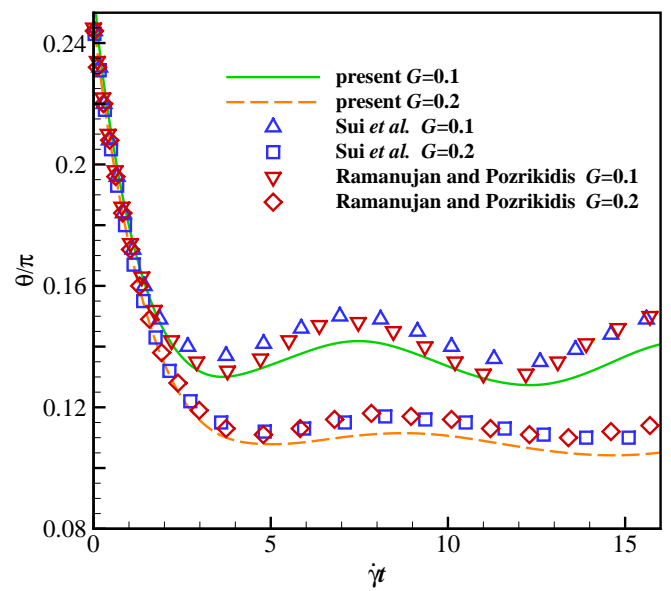

Figure 11: (a) The surface mesh of the oblate spherical capsule. (b) Time evolution of the Taylor deformation parameter $D_{x y}$ at $R e=0.025$ and $G=0.1$, and 0.2 . (c) Time evolution of the inclination $\theta$ at $R e=0.025$ and $G=0.1$, and 0.2 .

al. [96], and the experimental results by Zabusky et al. [97]. The present results agree well with previous numerical results for all $\Delta$ values considered, with all numerical studies diverging from the experimental results [97] for $\Delta>0.8$.

\subsection{A 3D flapping flag in a Newtonian free stream}

In this section, the case of a 3D flapping flag in a Newtonian free stream is presented to verify the model of the flexible flag. The schematic diagram of the problem is shown in Fig. 13. The computational domain is a rectangular box with dimensions $[-L, 7 L] \times[-2 L, 2 L] \times[-L, L]$. The square flag of length $L$ is initially flat, with the center of the leading edge positioned at the origin. The leading edge of the flag is pinned, and the other three edges are free to move. To speed up the onset of oscillatory motion, the flag is initially inclined at $\theta=0.1 \pi$ from the $x z$-plane, where $x$ is the streamwise direction and $z$ is tangential to the flag leading edge.

The simulation is performed on a uniform background volume grid with grid spacing $h=\Delta x=$ $\Delta y=\Delta z=L / 64$. Uniform surface grids with the same size are distributed on the flag. Dirichlet boundary conditions $\left(u_{x}=U, u_{y}=u_{z}=0\right)$ are applied to the inflow and boundaries in all lateral 


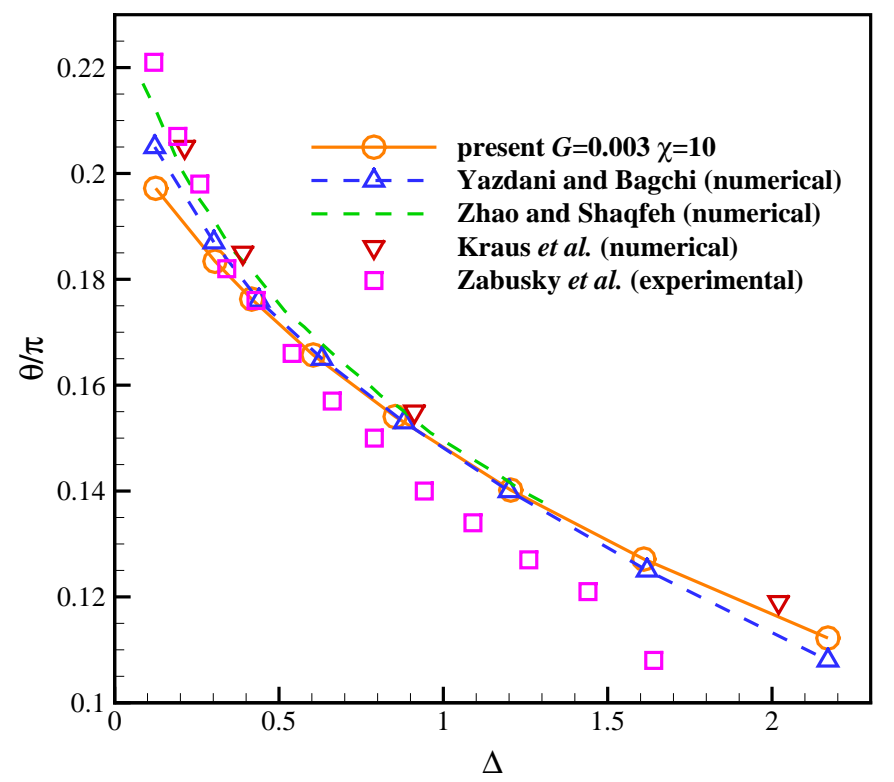

Figure 12: The dependence of inclination angles on the excess area $\Delta$ of the vesicle at $\widehat{\lambda}=1$ and $\chi=10$.

directions. The Neumann boundary condition $(\partial \boldsymbol{u} / \partial x=0)$ is applied at the outflow boundary $[98,99,100]$.

The mass ratio is $\rho_{s} /\left(\rho_{f} L\right)=1, \kappa_{11}^{T}=\kappa_{22}^{T}=10, \kappa_{12}^{T}=\kappa_{21}^{T}=1$, and $\kappa_{11}^{B}=\kappa_{22}^{B}=\kappa_{12}^{B}=0.0001$.

Fig. 14 shows the time histories of the transverse displacement of point $\mathrm{A}$ (as indicated in Fig. 13), the drag coefficient and the lift coefficient at $R e=U L / \nu=200$. The present results are compared with the numerical results of Huang and Sung [91], Tian et al. [101], de Tullio and Pascazio [102], and Lee and Choi [103]. Overall, the present results agree well with previous studies. We also observe obvious small discrepancies between each set of data, especially when the flag moves through the origin where friction drag is significant, and we believe this is caused by errors in predicting the friction. Specifically, first-order IBMs (e.g., Ref. [103] and present solver) predict a high friction, and thus a higher drag when the flag goes through the origin; while the second-order IBMs (e.g., Refs. [101, 102]) predict a lower friction leading to a low drag prediction. Note that the current method enables FSI problems involving viscoelastic fluids and the inertia of both fluids and structures, which will be further demonstrated in Section 5 .

\section{Applications}

In this section, the present method is applied to simulate the deformation of $2 \mathrm{D}$ and $3 \mathrm{D}$ capsules in an Oldroyd-B shear flow, a 3D flag flapping in an Oldroyd-B uniform flow, and the behaviours of 3D capsules in a contraction-expansion channel filled with an Oldroyd-B fluid to demonstrate the versatility of the method and to provide benchmark cases for the future development of FSI methods.

\subsection{The deformation of a $2 D$ circular capsule in an Oldroyd-B shear flow}

In this section, the present method is first applied to simulation of a $2 \mathrm{D}$ elastic capsule deformation in an Oldroyd-B shear flow. The configurations of the computational domain and the boundary 


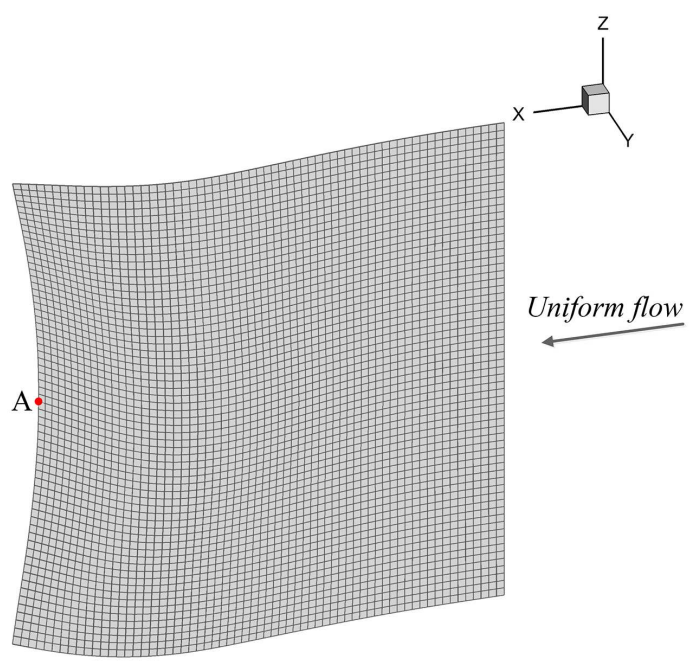

Figure 13: The schematic diagram of a 3D flag flapping in a Newtonian free stream.

conditions are the same as those used in Section 4.4 (See Fig. 9). The initial radius of the circular capsule is $R$, and the computational domain is $[0,16 R] \times[0,16 R]$. For simplicity, the fluids in the internal and external regions of the capsule are assumed to have the same density and viscosities (including solvent and polymer). The non-dimensional parameters are: $R e=\rho_{f} \dot{\gamma}(2 R)^{2} / \mu_{0}=0.05$, the Weissenberg number $W i=\lambda \dot{\gamma}$, the ratio of the solvent to the total viscosity $\beta=\mu_{s} / \mu_{0}=0.5$, the non-dimensional shear rate $G=\mu \dot{\gamma} R / k_{s}=0.04$, the non-dimensional diffusion parameter $\operatorname{Pr}=\kappa / \dot{\gamma}(2 R)^{2}=0.01$, and the non-dimensional bending modulus $e_{b}=k_{b} /\left(R^{2} k_{s}\right)$. The Taylor deformation parameter $D_{x y}$ and the inclination angle $\theta$ (see their definitions in Section 4.4) are used to characterise the deformation of the capsule. The grid spacing is $h=\Delta x=\Delta y=0.05 R$.

First, the effects of the viscoelasticity of the fluid on the capsule deformation are investigated. Fig. 15 shows the time evolution of $D_{x y}$ and $\theta$ at $e_{b}=0$ and $W i=0,0.25,0.5,1.0,2.0,10.0$, 50.0, and 100.0. It is observed that the steady-state value of $D_{x y}$ first decreases then increases with increasing $W i$, and the inclination angle decreases monotonically with increasing $W i$ for $W i \leq 2.0$, above which the short-time inclination becomes more variable. These phenomena are also observed in previous 3D numerical studies by Raffiee et al. [104]. Note that Raffiee et al. [104] did not consider the interplay of the viscoelastic effects of the internal and external liquids of the capsule. At higher values of $W i(W i>2)$, the viscoelasticity of the fluid seems to suppress the deformation of the capsule at first, and then to monotonically increase it.

Next, the effects of the bending resistance on the capsule deformation are studied. The simulations are conducted at $e_{b}=0,0.025,0.05,0.1$, and 0.2 and $W i=1.0$. Fig. 16 shows the time evolution of $D_{x y}$ and $\theta$. It is found that $D_{x y}$ experiences a monotonic decrease with increasing $e_{b}$, and $\theta$ increases with increasing $e_{b}$.

Finally, the effects of the artificial damping parameter $\kappa$ (in Eq. 37) on the dynamics of the capsule are investigated. Fig. 17 shows the time evolution of the Taylor deformation parameter $D_{x y}$ and the inclination angle $\theta$ at $W i=0.25,1.0$ and 10.0 and $\operatorname{Pr}=0.01,0.001$ and 0.0001 . It is observed that $\operatorname{Pr}$ has a very slight influence on the Taylor deformation parameter $D_{x y}$ and inclination angle $\theta$ at $W i=0.25$ if $\operatorname{Pr} W i \leq 0.0025$. In addition, $\operatorname{Pr}$ seems to have a stronger 
(a)

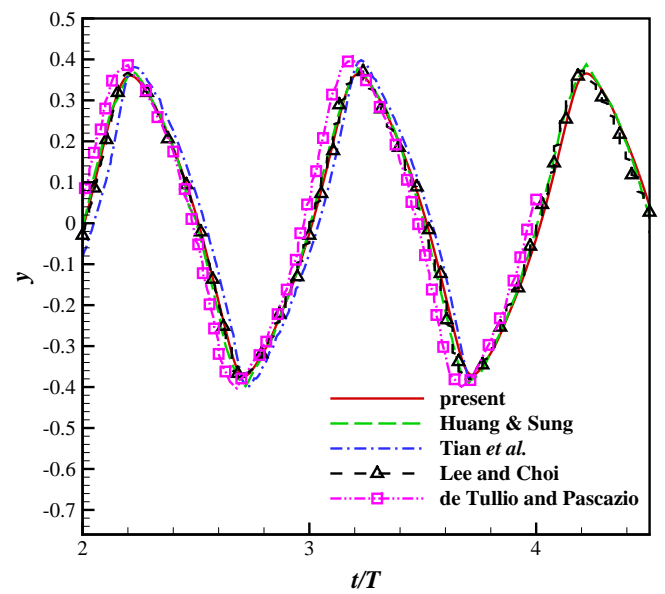

(b)

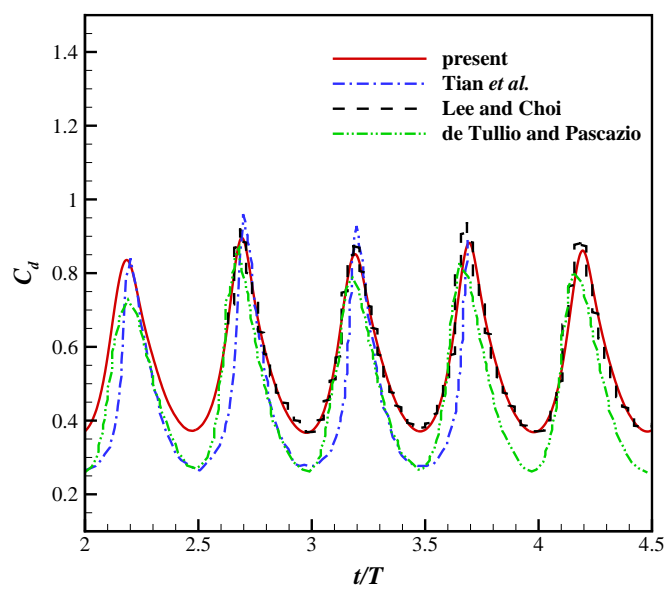

(c)

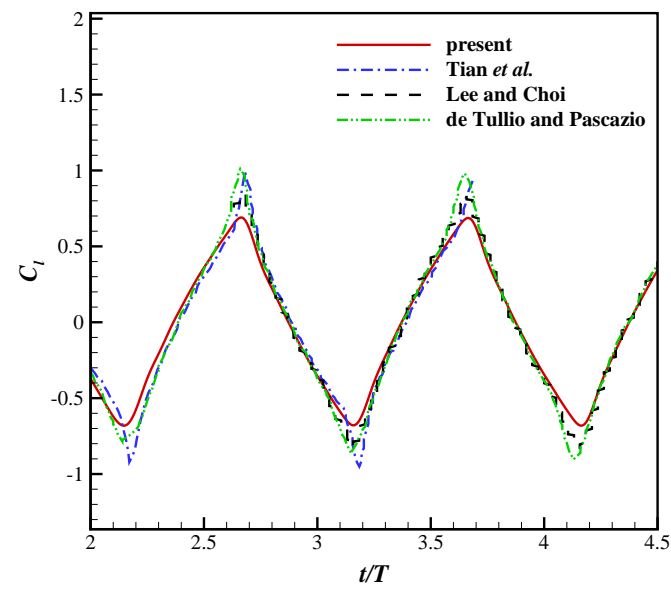

Figure 14: The time histories of (a) the trailing-edge transverse location (middle point), (b) the drag coefficient and (c) the lift coefficient of the flapping flag at $R e=200$.

influence on $D_{x y}$ and $\theta$ at $W i=1.0$ and 10.0 (especially at $\operatorname{Pr}=0.01$ ). However, the artificial damping parameter $\kappa$ does not significantly affect the dynamics of the capsule at $\operatorname{Pr} W i \leq O\left(10^{-3}\right)$. This is consistent with the conclusion obtained in Section 4.2.

\subsection{The deformation of a 3D spherical capsule with neo-Hookean law in an Oldroyd-B shear flow}

In this section, the present method is applied to 3D simulation of an initially spherical capsule (with NH law) deformation in an Oldroyd-B shear flow. The schematic diagram of the computational domain is shown in Fig. 7(a). The simulations are conducted in a cuboid domain of $[0,10 R] \times[0,10 R] \times[0,10 R]$, with the same boundary conditions as those in Section 4.2. The non-dimensional parameters are: the Reynolds number $R e=\rho_{f} \dot{\gamma} R^{2} / \mu_{0}=0.025$, the Weissenberg number $W i=\lambda \dot{\gamma}$, the ratio of the solvent to the total viscosity $\beta=\mu_{s} / \mu_{0}=0.5$, the nondimensional diffusion parameter $\operatorname{Pr}=\kappa / \dot{\gamma}(2 R)^{2}=6.25 \times 10^{-3}$, the non-dimensional shear rate $G=\mu_{0} \dot{\gamma} R / E_{s}=0.05$, and the non-dimensional bending stiffness $e_{b}=E_{b} /\left(R^{2} E_{s}\right)$. The internal and external fluids are also assumed to have the same properties (density and solvent and polymer 
(a)

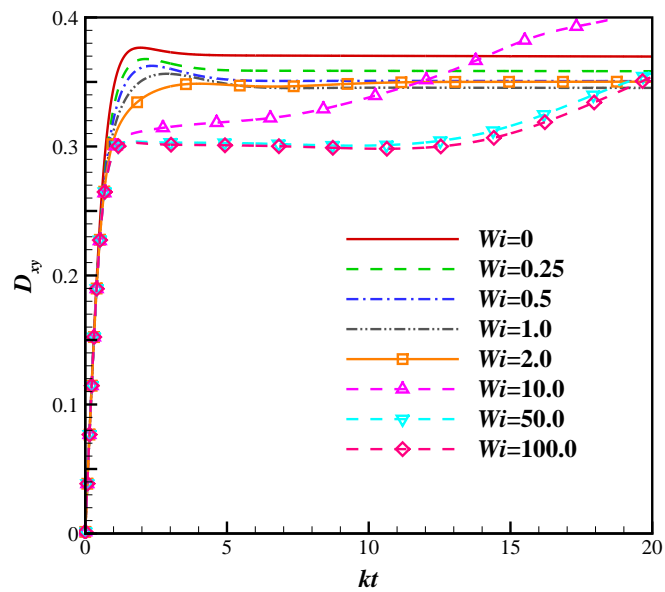

(b)

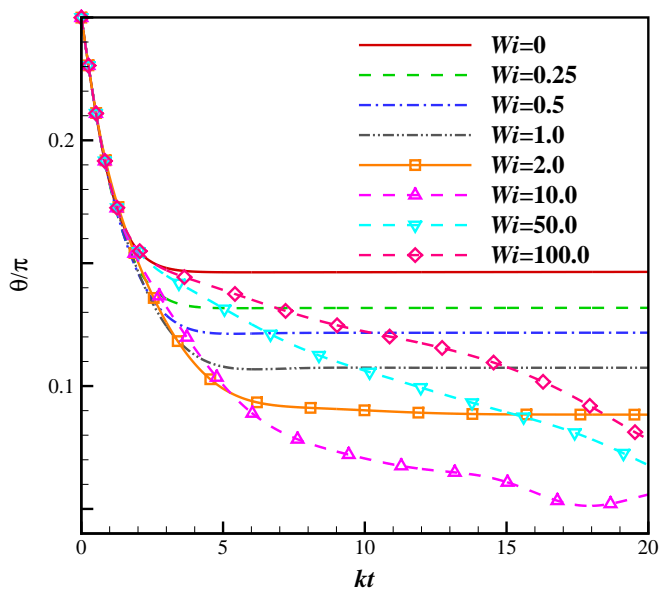

Figure 15: The temporal evolution of (a) Taylor deformation parameter and (b) inclination angle for $R e=0.05$, $G=0.04, e_{b}=0$ and different values of $W i$

(a)

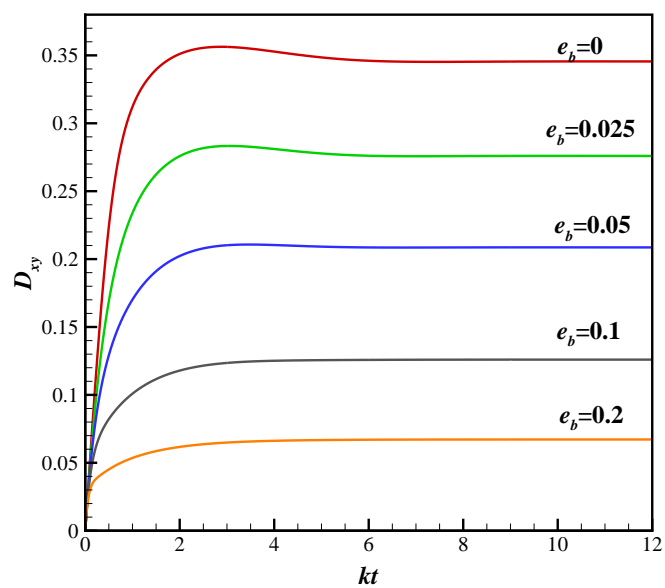

(b)

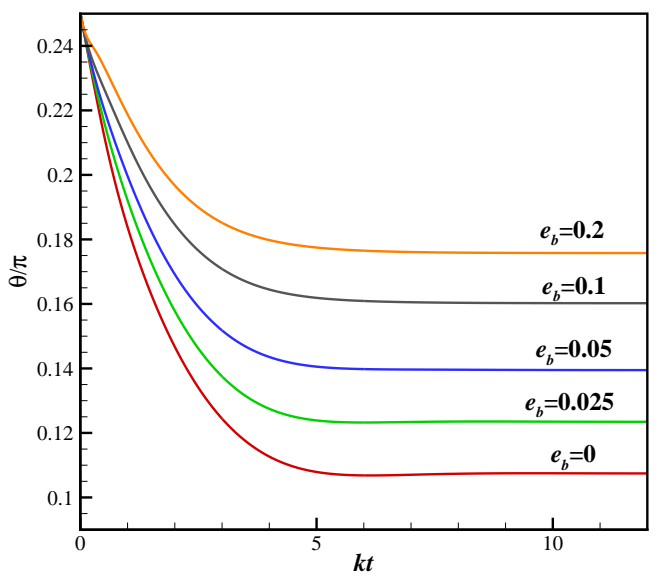

Figure 16: The temporal evolution of (a) Taylor deformation parameter and (b) inclination angle at $W i=1.0$, and different values of $e_{b}$.

viscosities). The grid spacing is $h=\Delta x=\Delta y=\Delta z=R / 12$.

First, the viscoelasticity of the fluid on the capsule deformation is studied, and the bending effects are neglected. The simulations are conducted at $W i=0,0.25,0.5,1.0,2.0,10.0$ and 100.0, and $e_{b}=0$. Fig. 18 shows the time history of $D_{x y}$ and $\theta$. It is observed that $D_{x y}$ attains a steady state value which decreases with increasing $W i$ for $W i \leq 1.0$. However, when $W i \geq 2.0$, $D_{x y}$ increases continuously and does not reach a steady state. This is due to buckling of the capsule, as shown in Fig. 19, where the length of the semi-major axes at plane $y=0$ decreases. Similar buckling behaviours have also been observed in Raffiee et al. [104], and are attributed to the nonlinearity of the Oldroyd-B fluid [104]. Note that the study of Raffiee et al. [104] did not consider the viscoelasticity of the fluid simultaneously for the internal and external regions of the 
(a)

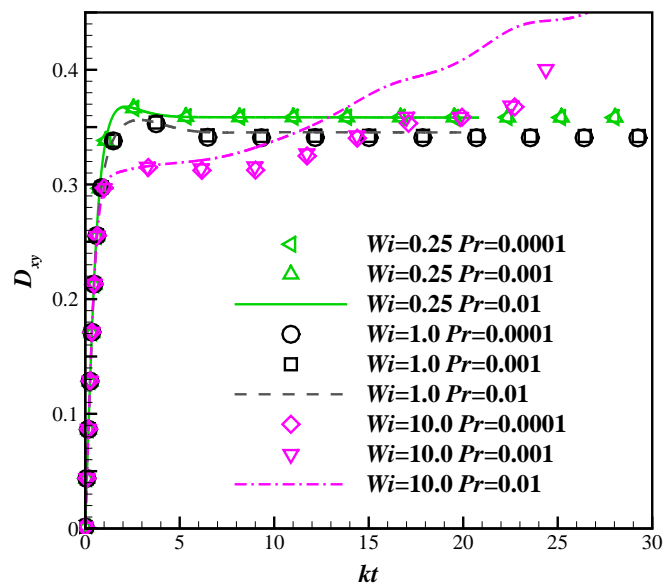

(b)

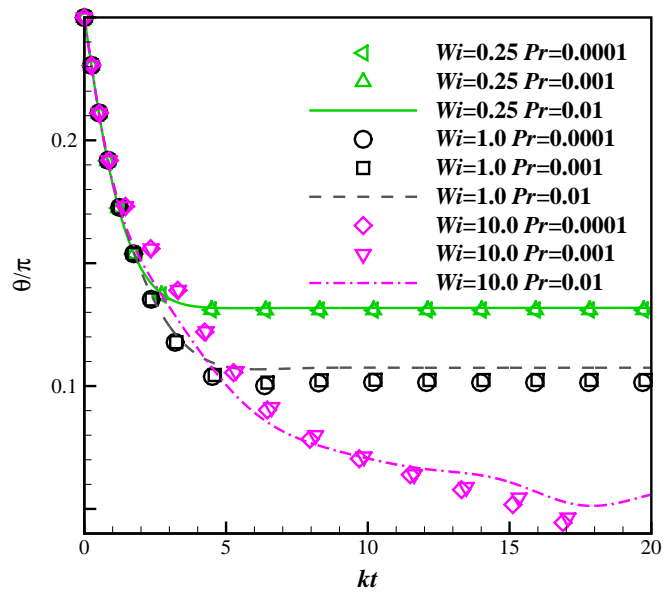

Figure 17: The temporal evolution of (a) Taylor deformation parameter and (b) inclination angle for $R e=0.05$, $G=0.04, e_{b}=0$ and different values of $W i$ and $\operatorname{Pr}$.

(a)

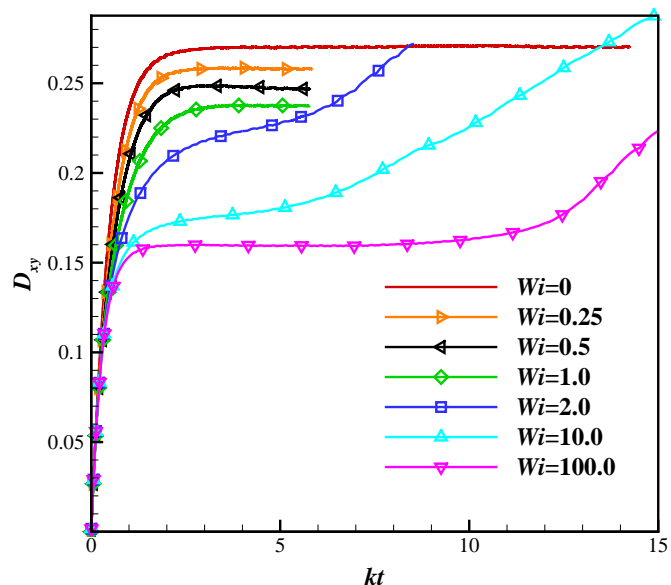

(b)

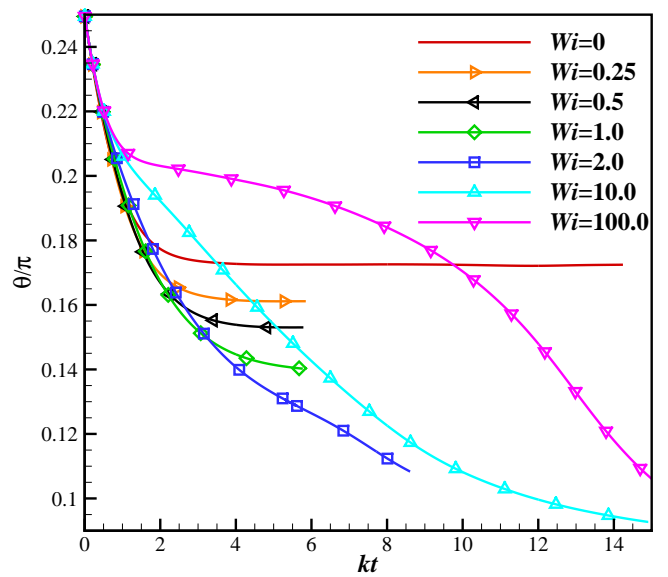

Figure 18: The temporal evolution of (a) Taylor deformation parameter and (b) inclination angle for different values of $W i$.

capsule. In addition, the steady inclination angle decreases with increasing $W i$ for $W i \leq 1.0$, and the inclination angle drops monotonically for $W i \geq 2.0$.

Next, the bending effects on the capsule deformation are investigated for $W i=1.0$ and $e_{b}=0$, $0.025,0.05,0.1$, and 0.2 . The time histories of $D_{x y}$ and $\theta$ are shown in Fig. 20. It is found that $D_{x y}$ decreases monotonically with increasing $e_{b}$, and the inclination angle increases with $e_{b}$.

As in Section 5.1, the effects of the artificial damping parameter $\kappa$ (in Eq. 37) on the dynamics of the capsule are investigated. Fig. 21 shows the time evolution of the Taylor deformation parameter $D_{x y}$ and the inclination angle $\theta$ at $W i=0.5,2.0$ and 10.0 and $\operatorname{Pr}=6.25 \times 10^{-3}, 6.25 \times 10^{-4}$ and $6.25 \times 10^{-5}$. Similar to the observation in Section 5.1, it is found that $\operatorname{Pr}$ does not have a significant influence on the Taylor deformation parameter and the inclination angle of the capsule when $\operatorname{Pr} W i \leq O\left(10^{-3}\right)\left(\operatorname{Pr} \leq 6.25 \times 10^{-3}\right.$ for $W i=0.5$, and $\operatorname{Pr} \leq 6.25 \times 10^{-4}$ for $W i=1.0$ and 
(a)

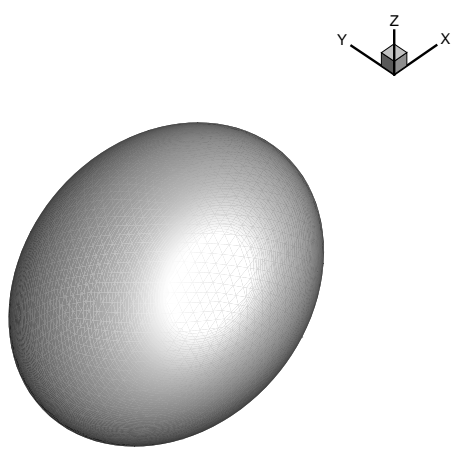

(c)

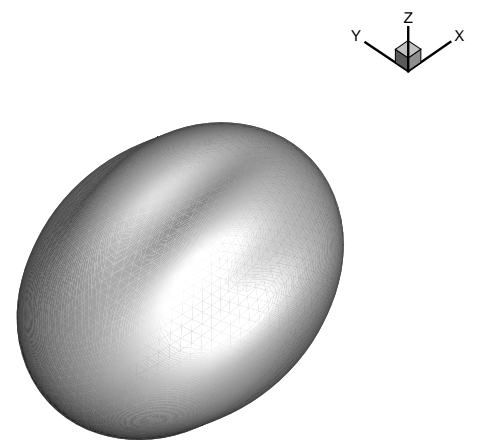

(b)

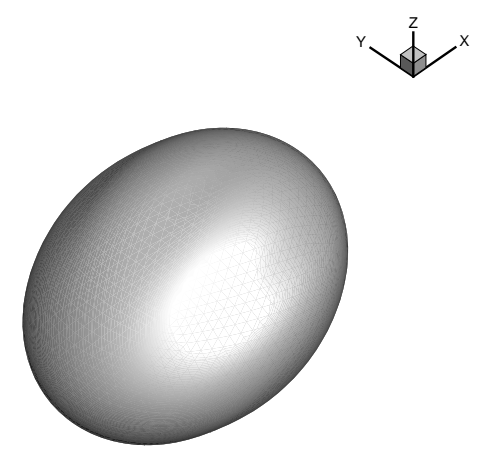

(d)

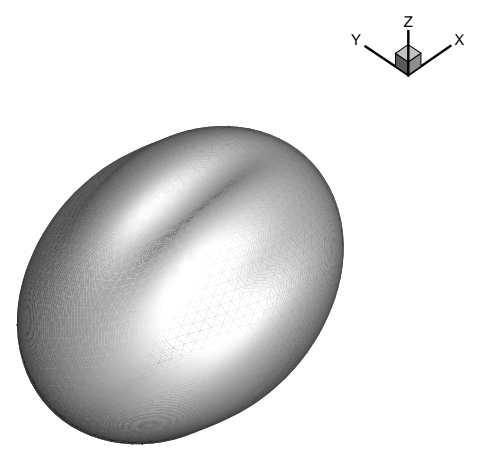

Figure 19: 3D profiles of the capsule at $W i=2.0$ at dimensionless time $k t=$ (a) 2, (b) 6, (c) 8, and (d) 10 .

$10.0)$.

\subsection{A 3D flag flapping in an Oldroyd-B free stream}

In this section, the present method is applied to simulate a 3D flag flapping in an Oldroyd-B free stream. The configurations of the fluid flow, the computational domain and the flag are identical to those used in Section 4.7. The non-dimensional parameters used in the simulations are $R e=$ $\rho_{f} U L / \mu_{0}=100$, the Weissenberg number $W i=\lambda U / L$, the ratio of the solvent to the total viscosity $\beta=\mu_{s} / \mu_{0}=0.5$, and the non-dimensional diffusion parameter constant $\operatorname{Pr}=\kappa /(U L)=1 \times 10^{-3}$.

Fig. 22(a) shows the time history of the transverse displacement of point $\mathrm{A}$ on the trailing edge on the flag at $W i$ ranging from 0 to 1.0 with an increment of 0.2 . It is observed that with the increase of $W i$ the flapping amplitude of the flag experiences monotonic decrease which means the viscoelasticity of the fluid hinders the flapping motion of the flag. In addition, to investigate the effects of the artificial damping parameter $\kappa$ (in Eq. 37) on FSI problems involving fluid and structural inertia, Fig. 22(b) shows the time history of the transverse displacement of point A at $W i=1.0$ and different $\operatorname{Pr}$. It is found that $\operatorname{Pr}$ significantly affects the transverse displacement of 
(a)

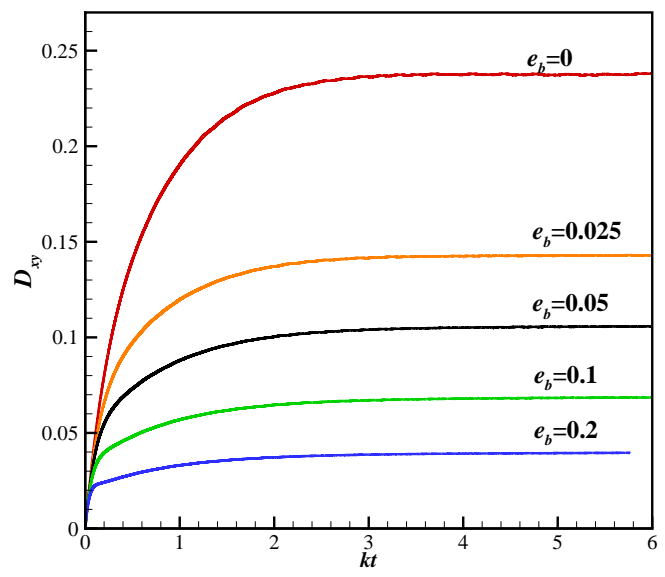

(b)

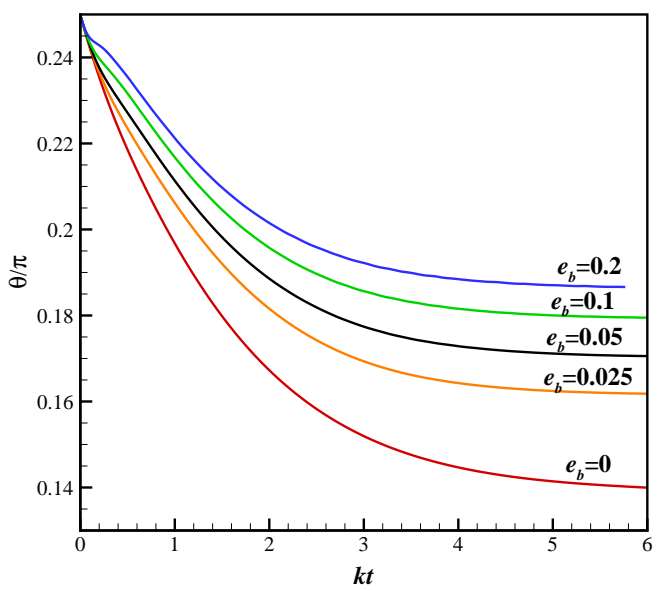

Figure 20: The temporal evolution of (a) Taylor deformation parameter and (b) inclination angle for $W i=1.0$ and different values of $e_{b}$.

(a)

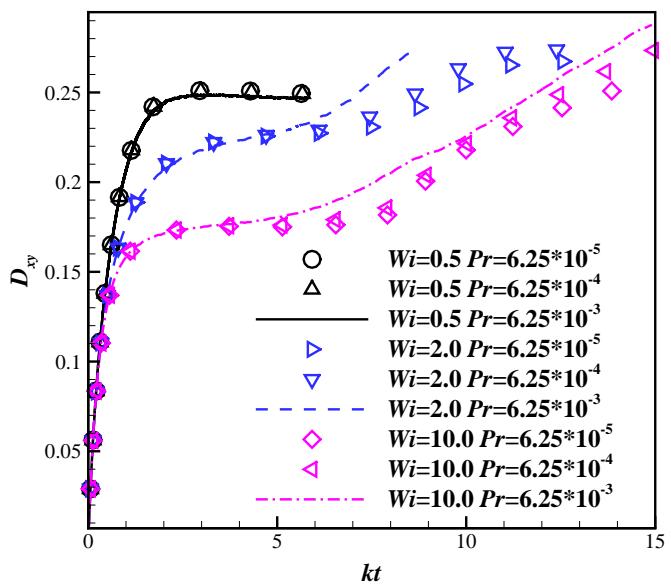

(b)

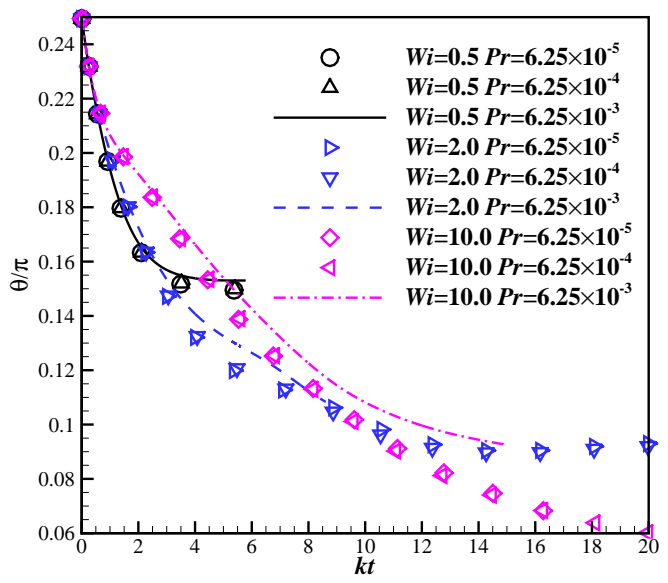

Figure 21: The temporal evolution of (a) Taylor deformation parameter and (b) inclination angle for different values of $W i$ and $P r$.

point A when $\operatorname{Pr} W i>1 \times 10^{-3}\left(\operatorname{Pr}=1 \times 10^{-2}\right.$ and $\left.1 \times 10^{-1}\right)$ compared with the slight influence when $\operatorname{Pr} W i \leq 1 \times 10^{-3}\left(\operatorname{Pr}=1 \times 10^{-3}\right.$ and $\left.1 \times 10^{-4}\right)$.

To provide data for future validation of newly developed methods, Figs. 23, 24, and 25 show the snapshots of the vortical structures (characterized by the isosurface using the $Q$-criterion $[101,105]$ ) for nine time instants as indicated in Fig. 22. In addition, Figs. 26-31 show the isosurface for the first invariant of stress tensor $\left(D_{1}\right)$ and the second invariant of stress tensor $\left(D_{2}\right)$ at the same instants. 
(a)

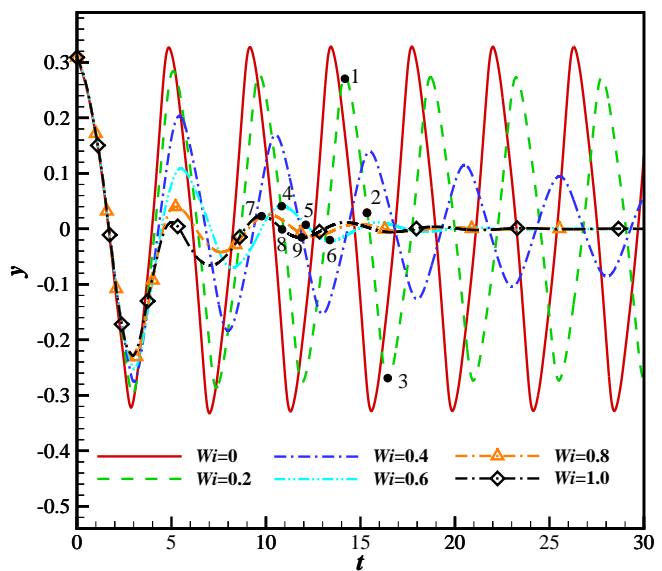

(b)

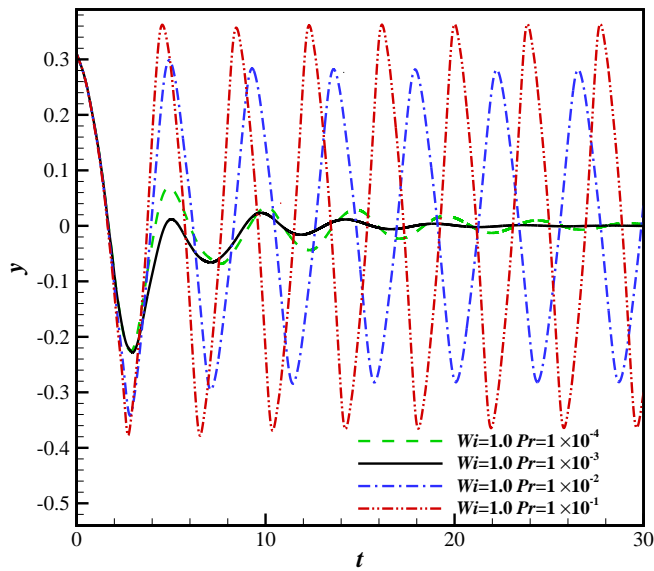

Figure 22: (a) The time history of the trailing-edge transverse location (middle point) of the flapping flag in the Oldroyd-B fluid uniform flow for $R e=100$ and different values of $W i$. Numbers 1-9 indicate time instants for visualizations in Figs. 23 to 31. (b) The effects of $\operatorname{Pr}$ on the time history of the trailing-edge transverse location (middle point) of the flapping flag.

(a)

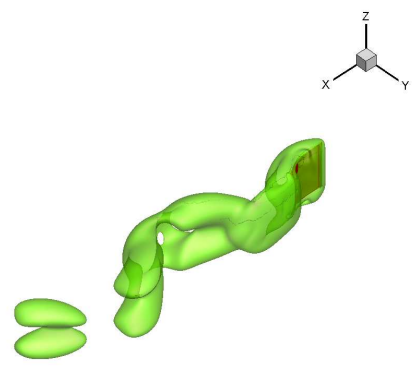

(b)

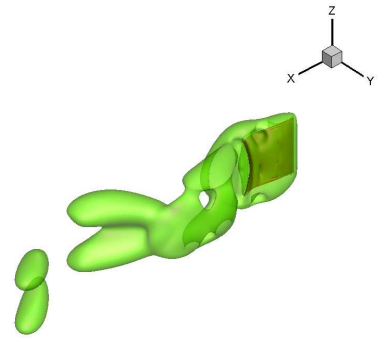

(c)

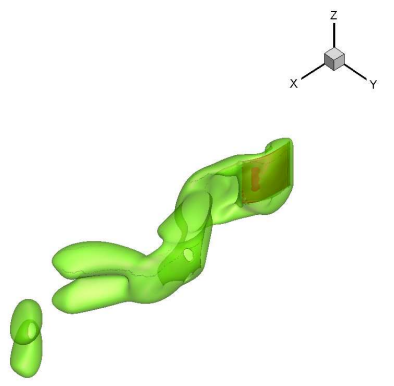

Figure 23: The vortex structure shedding from the flapping flag for $W i=0.2$ at $t=14.177$ (instant 1 ), 15.309 (instant 2), and 16.442 (instant 3).

\subsection{The behaviours of $3 D$ capsules in a contraction-expansion channel filled with the Oldroyd-B fluid}

To demonstrate the capability of the present method for FSI problems involving complicated geometries, the behaviours of 12 initially spherical capsules described by Skalak's law in a contractionexpansion channel filled with Oldroyd-B fluid are studied in this section. The schematic diagram of the problem is shown in Fig. 32. The internal and external fluids of the capsules have the same properties (density and solvent and polymer viscosities). The initial radius of the capsules is $R$, and the computational domain is $[0, L] \times[0, W] \times[0, H]$ with $L=25 R, W=6 R$ and $H$ $=10 R$. The contraction segment is located at $[10,15 R] \times[0, W] \times[3 R, 10 R]$. The fluid is assumed to be driven by a pressure gradient $d p / d x$ along the channel in the positive $x$-direction, which is achieved by implementing a constant body force as $f_{x}=-d p / d x$. Periodic boundary conditions are applied to the inlet and outlet of the channel. The non-dimensional parameters 
(a)

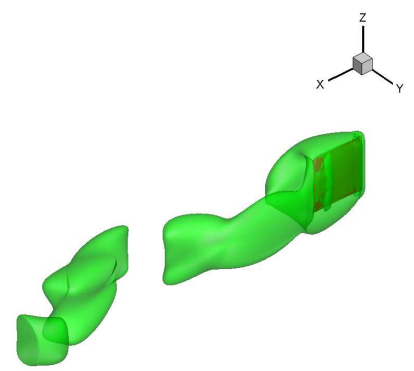

(b)

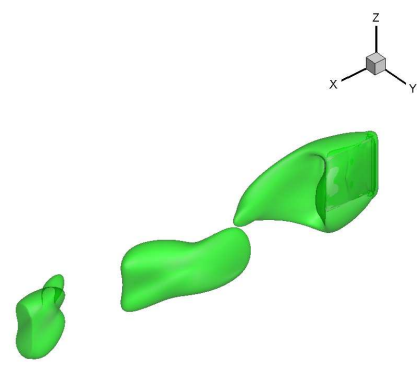

(c)

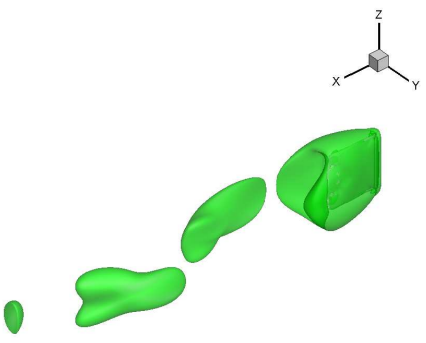

Figure 24: The vortex structure shedding from the flapping flag for $W i=0.6$ at $t=10.832$ (instant 4), 12.106 (instant 5), and 13.378 (instant 6).

(a)

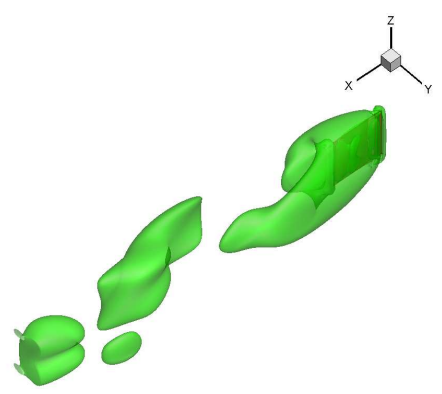

(b)

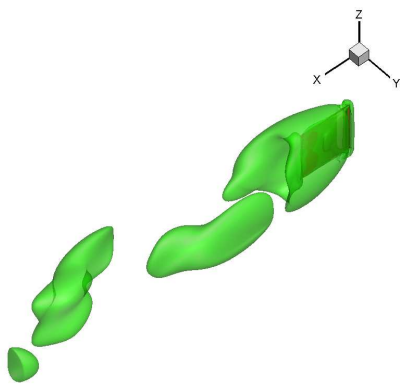

(c)

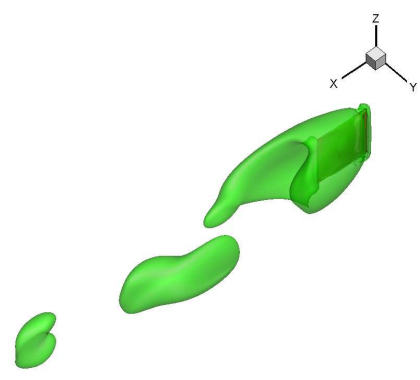

Figure 25: The vortex structure shedding from the flapping flag for $W i=1.0$ at $t=9.766$ (instant 7), 10.852 (instant 8), and 11.884 (instant 9).

(a)

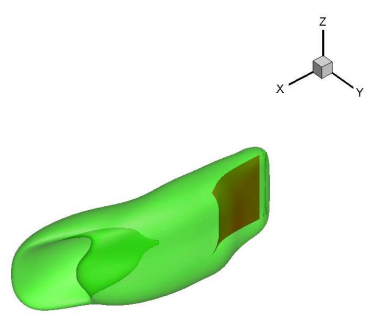

(b)

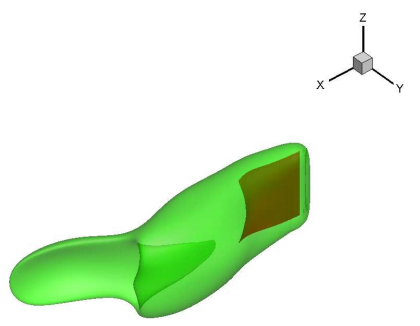

(c)<smiles></smiles>

Figure 26: The iso-surface of $D_{1}$ for the flapping flag for $W i=0.2$ at $t=14.177$ (instant 1), 15.309 (instant 2), and 16.442 (instant 3). 
(a)

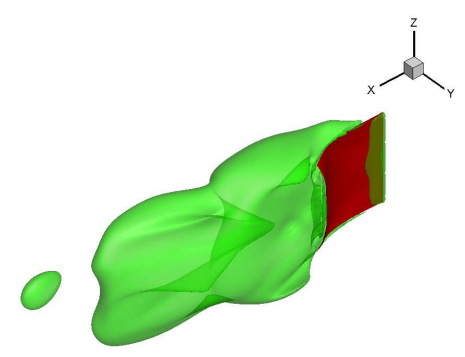

(b)

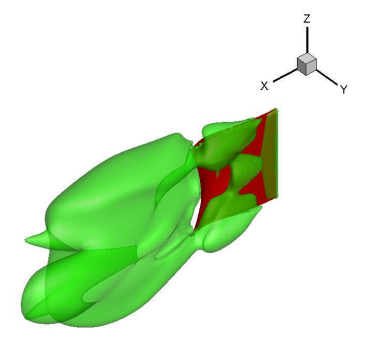

(c)

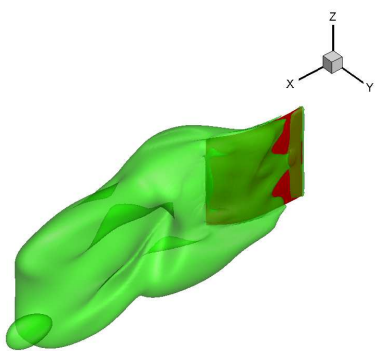

Figure 27: The iso-surface of $D_{2}$ for the flapping flag for $W i=0.2$ at $t=14.177$ (instant 1), 15.309 (instant 2), and 16.442 (instant 3).

(a)

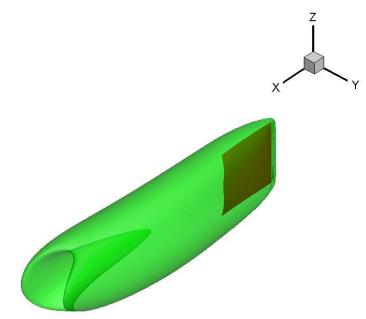

(b)

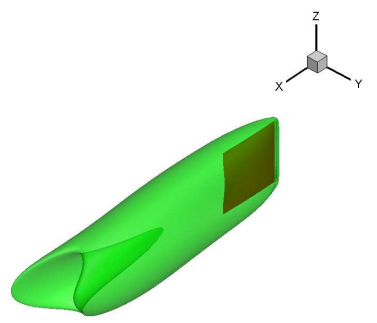

(c)

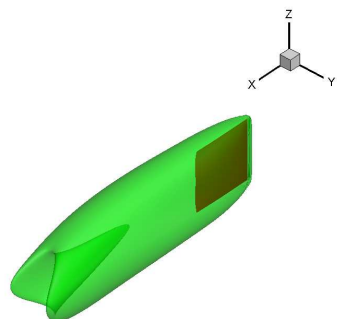

Figure 28: The iso-surface of $D_{1}$ for the flapping flag for $W i=0.6$ at $t=10.832$ (instant 4), 12.106 (instant 5 ), and 13.378 (instant 6).

(a)

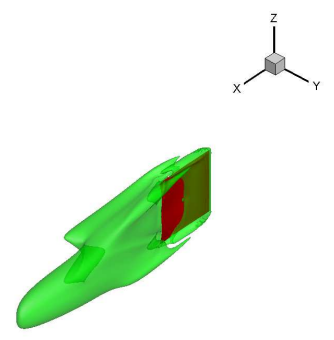

(b)

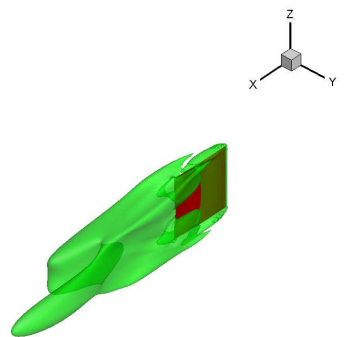

(c)<smiles></smiles>

Figure 29: The iso-surface of $D_{2}$ for the flapping flag for $W i=0.6$ at $t=10.832$ (instant 4), 12.106 (instant 5), and 13.378 (instant 6). 
(a)

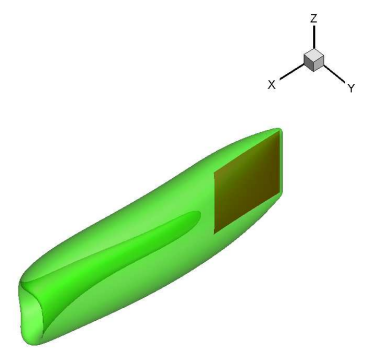

(b)

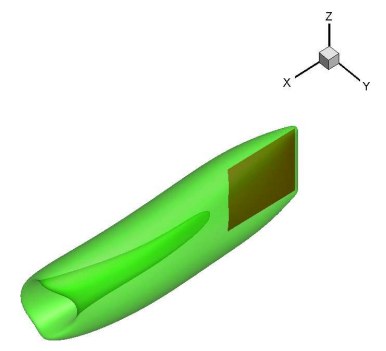

(c)

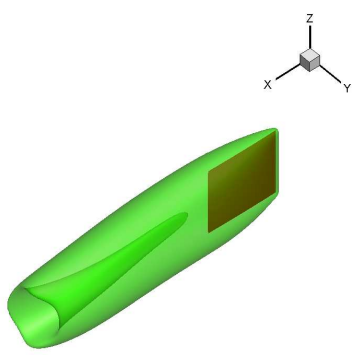

Figure 30: The iso-surface of $D_{1}$ for the flapping flag for $W i=1.0$ at $t=9.766$ (instant 7), 10.852 (instant 8), and 11.884 (instant 9).

(a)

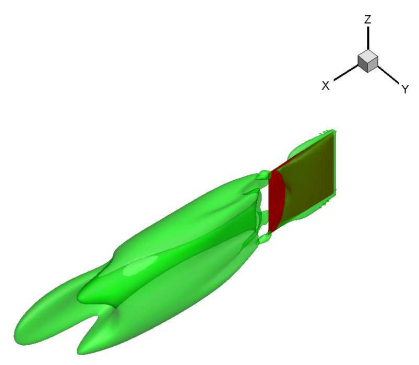

(b)

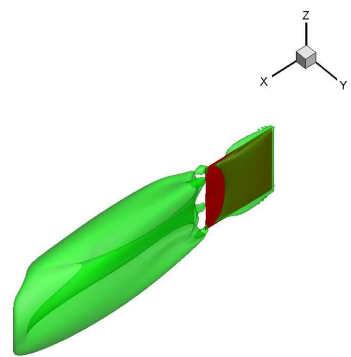

(c)

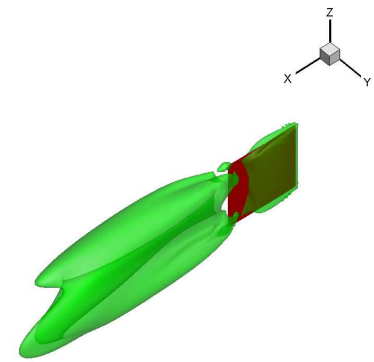

Figure 31: The iso-surface of $D_{2}$ for the flapping flag for $W i=1.0$ at $t=9.766$ (instant 7), 10.852 (instant 8), and 11.884 (instant 9).

are defined as: $R e=\rho_{f} U_{c} L_{c} / \mu_{0}=100$, the Weissenberg number $W i=\lambda U_{c} / L_{c}=0.5$, the ratio of the solvent to the total viscosity $\beta=\mu_{s} / \mu_{0}=0.5$, the non-dimensional diffusion parameter $\operatorname{Pr}=\kappa /\left(\dot{\gamma} L_{c}^{2}\right)=1 \times 10^{-3}$, the capillary number $C a=\mu_{0} U_{c} / E_{s}=0.1$, and the non-dimensional bending stiffness $e_{b}=E_{b} /\left(R^{2} E_{s}\right)=0.0008$, where $U_{c}=W^{2} f_{x} /\left(8 \mu_{0}\right), L_{c}$ is the hydraulic diameter of the channel, defined as $\frac{2 W H}{W+H}$, and $\dot{\gamma}=\frac{2 U_{c}}{W}$ is the wall shear rate. The elastic modulus for area dilation (in Eq. 15) used in the simulations is $E_{a}=2 E_{s}$. The grid spacing is $h=\Delta x=\Delta y=\Delta z=R / 10$.

Fig. 33 shows the time evolution of the capsule configuration at non-dimensional time $t=0,20$, $44,71,92,109.667,154$, and 181.667. It is found that the capsules (initially placed in the channel orderly) mix up after a short-term evolution, and then migrate to the bottom of the channel and almost follow two steady trajectories after a long-term evolution (see Fig. 34).

\section{Conclusion}

We have presented a numerical method for FSI problems involving viscoelastic fluids and complex geometries. In this method, the fluid dynamics and the constitutive equations of viscoelastic 


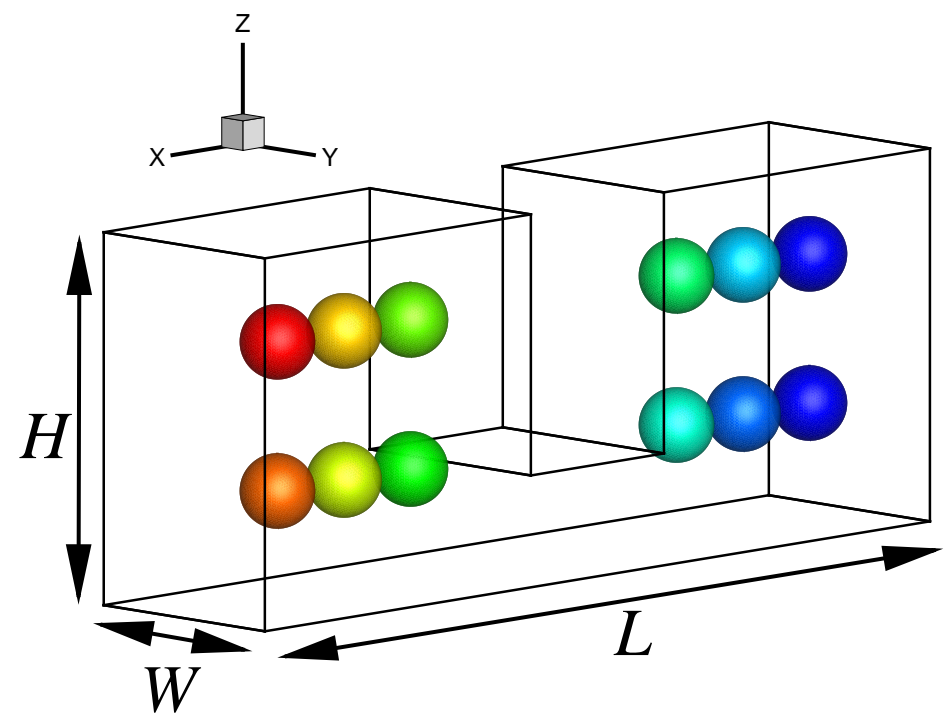

Figure 32: The schematic diagram of the behaviours of 12 capsules in the contraction-expansion channel. The colours are used to mark capsules.

fluids are both solved using the lattice Boltzmann method. The interaction between the solid structure and the fluid is achieved by the immersed boundary method. The structure equations are solved by using the finite element method (3D capsules) and the finite difference method (2D capsules, 2D and 3D rigid particles, and flags). The present method and structure models are validated by several cases including a 2D rigid particle migration in a Giesekus Couette flow, a spherical particle rotation in an Oldroyd-B shear flow, a spherical particle settling in a FENE-CR fluid, 2D and 3D capsules deformation in a Newtonian shear flow, and a 3D flag flapping in a Newtonian free stream. The numerical results are successfully validated against previous experimental and numerical results. In addition, the present method is also applied to simulate 2D and 3D capsule deformation in an Oldroyd-B shear flow, a 3D flag flapping in an Oldroyd-B free stream, and the behaviours of 3D capsules in a contraction-expansion channel filled with an Oldroyd-B fluid. For capsules in an Oldroyd-B fluid shear flow, we find that the capsules experience smaller deformation at larger Weissenberg numbers, and the capsules experience monotonically increasing deformation when the Weissenberg number is above a critical value which is 10 for $2 \mathrm{D}$ and 2 for $3 \mathrm{D}$ simulations. For a 3D flag flapping in an Oldroyd-B free stream, the viscoelasticity of the fluid hinders the flapping motion of the flag. For elastic capsule migration in a contraction-expansion channel, the capsules form two lines after a long-term evolution. The overall framework of this method is very simple, and allows us to tackle FSI problems involving both the fluid and structure inertia as well as the viscoelastic fluids without any preconditioner. Our validations and applications show that this method is very efficient for a large ranges of Weissengberg and Reynolds numbers. The results 
(a)

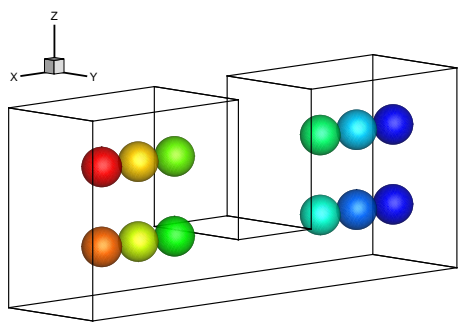

Time $=0$

(c)

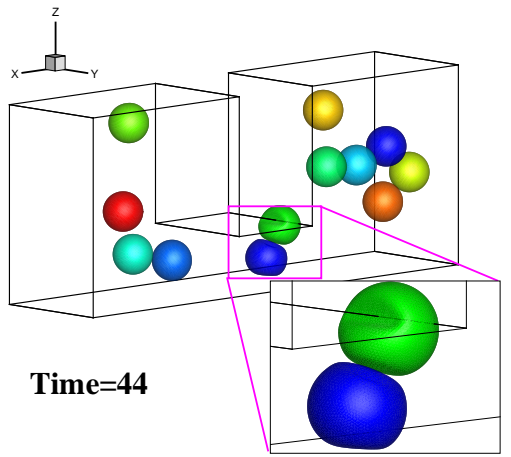

(e)

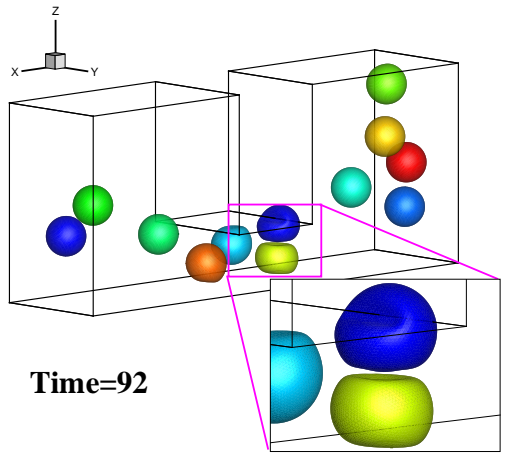

(g)

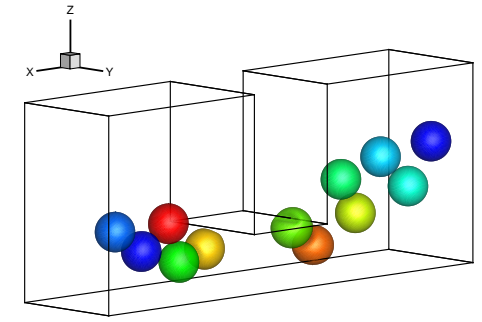

Time=154 (b)

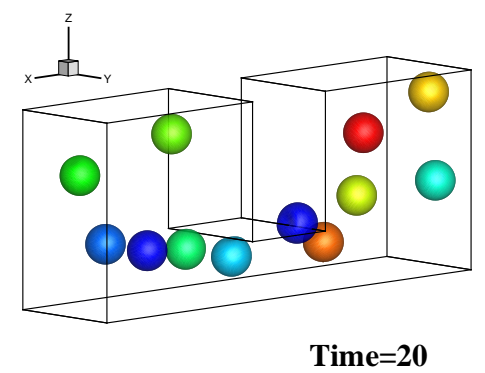

(d)

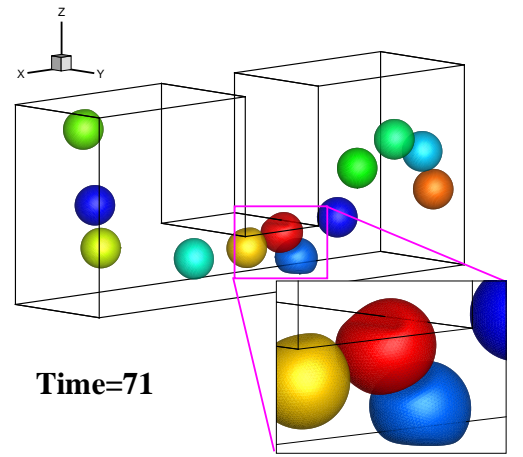

(f)

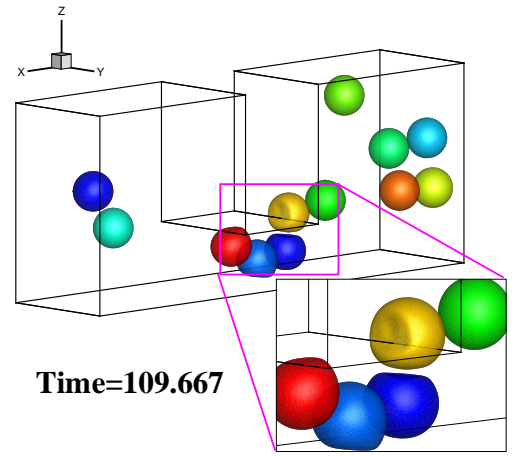

(h)

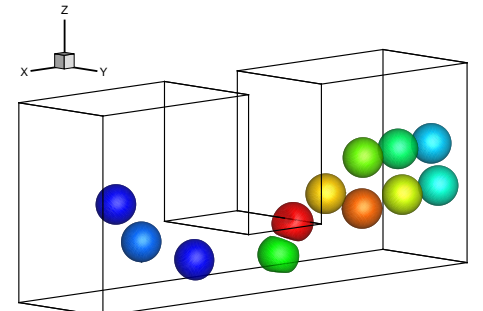

Time $=181.667$

Figure 33: The configuration of 12 elastic capsules in the contraction-expansion channel at $t=$ (a) 0 , (b) 20, (c) 44, (d) 71, (e) 92, (f) 109.667, (g) 154, and (h) 181.667. 

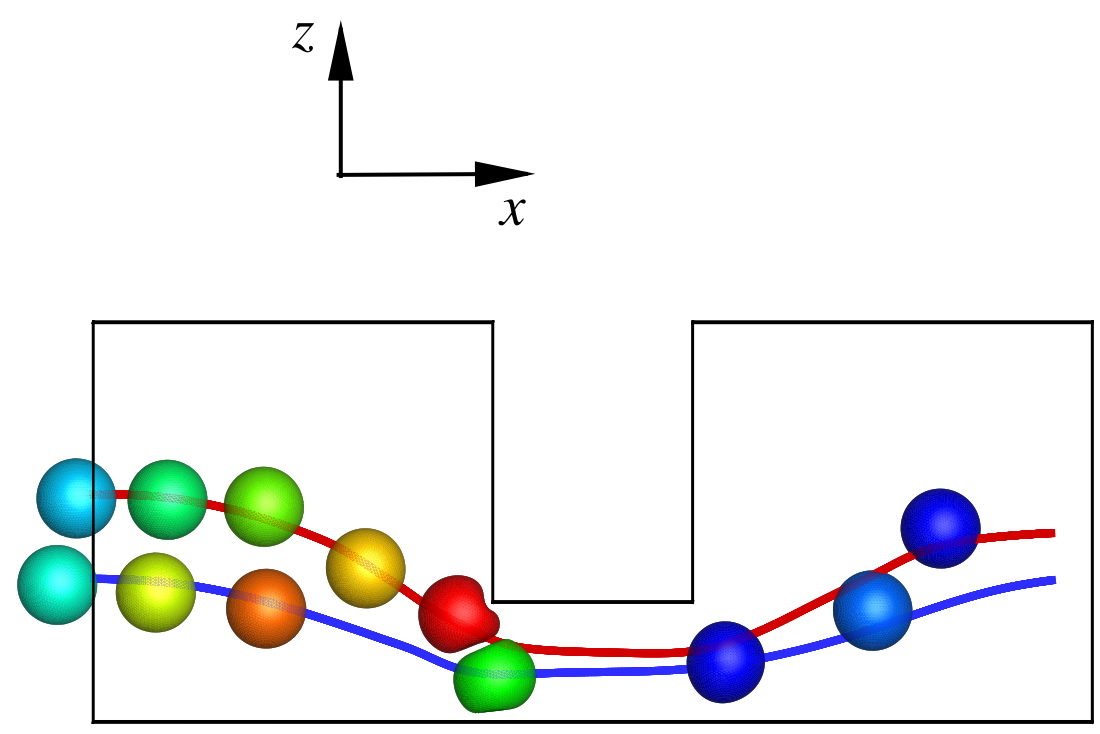

Time $=181.667$

Figure 34: The configuration of 12 elastic capsules in the contraction-expansion channel at $t=181.667$ in $x-z$ plane. Red line: the steady trajectory of the upper capsules follow. Blue line: the steady trajectory of the lower capsules follow.

provide well documented benchmarks expanding the currently limited data base of FSI benchmark study.

\section{Acknowledgements}

This research is undertaken with the assistance of resources from the National Computational Infrastructure, which is supported by the Australian Government. J. MA acknowledges the UNSW University College Postgraduate Research Scholarship during the pursuit of this study. F.-B. Tian is the recipient of an Australian Research Council Discovery Early Career Researcher Award (project number DE160101098).

\section{References}

[1] H. Guo, E. Kanso, A computational study of mucociliary transport in healthy and diseased environments, European Journal of Computational Mechanics 26 (2017) 4-30.

[2] J. Feng, P. Huang, D. Joseph, Dynamic simulation of sedimentation of solid particles in an Oldroyd-B fluid, Journal of Non-Newtonian Fluid Mechanics 63 (1996) 63-88.

[3] A. Groisman, V. Steinberg, Elastic turbulence in a polymer solution flow, Nature 405 (2000) 53. 
[4] Y. J. Liu, J. Nelson, J. Feng, D. D. Joseph, Anomalous rolling of spheres down an inclined plane, Journal of Non-Newtonian Fluid Mechanics 50 (1993) 305-329.

[5] X. Lu, L. Zhu, R.-m. Hua, X. Xuan, Continuous sheath-free separation of particles by shape in viscoelastic fluids, Applied Physics Letters 107 (2015) 264102.

[6] X. Lu, X. Xuan, Elasto-inertial pinched flow fractionation for continuous shape-based particle separation, Analytical Chemistry 87 (2015) 11523-11530.

[7] D. Yuan, S. H. Tan, Q. Zhao, S. Yan, R. Sluyter, N.-T. Nguyen, J. Zhang, W. Li, Sheathless dean-flow-coupled elasto-inertial particle focusing and separation in viscoelastic fluid, RSC Advances 7 (2017) 3461-3469.

[8] G. Holzner, S. Stavrakis, A. DeMello, Elasto-inertial focusing of mammalian cells and bacteria using low molecular, low viscosity PEO solutions, Analytical Chemistry 89 (2017) 1165311663.

[9] M. A. Faridi, H. Ramachandraiah, I. Banerjee, S. Ardabili, S. Zelenin, A. Russom, Elastoinertial microfluidics for bacteria separation from whole blood for sepsis diagnostics, Journal of Nanobiotechnology 15 (2017) 3.

[10] X. Lu, C. Liu, G. Hu, X. Xuan, Particle manipulations in non-Newtonian microfluidics: A review, Journal of Colloid and Interface Science 500 (2017) 182-201.

[11] E. J. Lim, T. J. Ober, J. F. Edd, S. P. Desai, D. Neal, K. W. Bong, P. S. Doyle, G. H. McKinley, M. Toner, Inertio-elastic focusing of bioparticles in microchannels at high throughput, Nature Communications 5 (2014) 4120.

[12] C. Liu, B. Ding, C. Xue, Y. Tian, G. Hu, J. Sun, Sheathless focusing and separation of diverse nanoparticles in viscoelastic solutions with minimized shear thinning, Analytical Chemistry 88 (2016) 12547-12553.

[13] D. Yuan, Q. Zhao, S. Yan, S.-Y. Tang, G. Alici, J. Zhang, W. Li, Recent progress of particle migration in viscoelastic fluids, Lab on a Chip (2018).

[14] G. D'Avino, F. Greco, P. L. Maffettone, Particle migration due to viscoelasticity of the suspending liquid and its relevance in microfluidic devices, Annual Review of Fluid Mechanics 49 (2017) 341-360.

[15] L. Becker, G. McKinley, H. K. Rasmussen, O. Hassager, The unsteady motion of a sphere in a viscoelastic fluid, Journal of Rheology 38 (1994) 377-403.

[16] C. Bodart, M. J. Crochet, The time-dependent flow of a viscoelastic fluid around a sphere, Journal of Non-Newtonian Fluid Mechanics 54 (1994) 303-329.

[17] X. Lu, J.-P. Hsu, X. Xuan, Exploiting the wall-induced non-inertial lift in electrokinetic flow for a continuous particle separation by size, Langmuir 31 (2014) 620-627.

[18] G. D'Avino, G. Cicale, M. Hulsen, F. Greco, P. Maffettone, Effects of confinement on the motion of a single sphere in a sheared viscoelastic liquid, Journal of Non-Newtonian Fluid Mechanics 157 (2009) 101-107. 
[19] J. Hao, T.-W. Pan, R. Glowinski, D. D. Joseph, A fictitious domain/distributed lagrange multiplier method for the particulate flow of Oldroyd-B fluids: A positive definiteness preserving approach, Journal of Non-Newtonian Fluid Mechanics 156 (2009) 95-111.

[20] M. Hashemi, R. Fatehi, M. Manzari, SPH simulation of interacting solid bodies suspended in a shear flow of an Oldroyd-B fluid, Journal of Non-Newtonian Fluid Mechanics 166 (2011) $1239-1252$.

[21] N. Goyal, J. Derksen, Direct simulations of spherical particles sedimenting in viscoelastic fluids, Journal of Non-Newtonian Fluid Mechanics 183 (2012) 1-13.

[22] G. Li, G. H. McKinley, A. M. Ardekani, Dynamics of particle migration in channel flow of viscoelastic fluids, Journal of Fluid Mechanics 785 (2015) 486-505.

[23] F. Snijkers, G. D'Avino, P. Maffettone, F. Greco, M. Hulsen, J. Vermant, Effect of viscoelasticity on the rotation of a sphere in shear flow, Journal of Non-Newtonian Fluid Mechanics 166 (2011) 363-372.

[24] Y. K. Lee, K. H. Ahn, A novel lattice Boltzmann method for the dynamics of rigid particles suspended in a viscoelastic medium, Journal of Non-Newtonian Fluid Mechanics 244 (2017) $75-84$.

[25] Z. Y. Luo, B. F. Bai, Dynamics of capsules enclosing viscoelastic fluid in simple shear flow, Journal of Fluid Mechanics 840 (2018) 656-687.

[26] M. A. Hulsen, R. Fattal, R. Kupferman, Flow of viscoelastic fluids past a cylinder at high Weissenberg number: stabilized simulations using matrix logarithms, Journal of NonNewtonian Fluid Mechanics 127 (2005) 27-39.

[27] R. Fattal, R. Kupferman, Time-dependent simulation of viscoelastic flows at high Weissenberg number using the log-conformation representation, Journal of Non-Newtonian Fluid Mechanics 126 (2005) 23-37.

[28] N. Balci, B. Thomases, M. Renardy, C. R. Doering, Symmetric factorization of the conformation tensor in viscoelastic fluid models, Journal of Non-Newtonian Fluid Mechanics 166 (2011) 546-553.

[29] X. Chen, H. Marschall, M. Schäfer, D. Bothe, A comparison of stabilisation approaches for finite-volume simulation of viscoelastic fluid flow, International Journal of Computational Fluid Dynamics 27 (2013) 229-250.

[30] P. Huang, J. Feng, H. H. Hu, D. D. Joseph, Direct simulation of the motion of solid particles in Couette and Poiseuille flows of viscoelastic fluids, Journal of Fluid Mechanics 343 (1997) 73-94.

[31] M. M. Villone, F. Greco, M. A. Hulsen, P. L. Maffettone, Numerical simulations of deformable particle lateral migration in tube flow of Newtonian and viscoelastic media, Journal of NonNewtonian Fluid Mechanics 234 (2016) 105-113. 
[32] M. Villone, F. Greco, M. Hulsen, P. Maffettone, Simulations of an elastic particle in Newtonian and viscoelastic fluids subjected to confined shear flow, Journal of Non-Newtonian Fluid Mechanics 210 (2014) 47-55.

[33] P. Yue, C. Zhou, J. J. Feng, C. F. Ollivier-Gooch, H. H. Hu, Phase-field simulations of interfacial dynamics in viscoelastic fluids using finite elements with adaptive meshing, Journal of Computational Physics 219 (2006) $47-67$.

[34] P. Yue, J. J. Feng, C. A. Bertelo, H. H. Hu, An arbitrary Lagrangian-Eulerian method for simulating bubble growth in polymer foaming, Journal of Computational Physics 226 (2007) $2229-2249$.

[35] F.-B. Tian, R. P. Bharti, Y.-Q. Xu, Deforming-spatial-domain/stabilized space-time (DSD/SST) method in computation of non-Newtonian fluid flow and heat transfer with moving boundaries, Computational Mechanics 53 (2014) 257-271.

[36] T. Tezduyar, M. Behr, J. Liou, A new strategy for finite element computations involving moving boundaries and interfacesthe deforming-spatial-domain/space-time procedure: I. the concept and the preliminary numerical tests, Computer Methods in Applied Mechanics and Engineering 94 (1992) 339 - 351.

[37] T. E. Tezduyar, M. Behr, S. Mittal, J. Liou, A new strategy for finite element computations involving moving boundaries and interfacesthe deforming-spatial-domain/space-time procedure: Ii. computation of free-surface flows, two-liquid flows, and flows with drifting cylinders, Computer Methods in Applied Mechanics and Engineering 94 (1992) 353-371.

[38] T. E. Tezduyar, Stabilized finite element formulations for incompressible flow computations, in: Advances in Applied Mechanics, volume 28, Elsevier, 1991, pp. 1-44.

[39] F.-B. Tian, Y. Wang, J. Young, J. C. Lai, An FSI solution technique based on the DSD/SST method and its applications, Mathematical Models and Methods in Applied Sciences 25 (2015) 2257-2285.

[40] T. E. Tezduyar, Computation of moving boundaries and interfaces and stabilization parameters, International Journal for Numerical Methods in Fluids 43 (2003) 555-575.

[41] S.-H. Chiu, T.-W. Pan, R. Glowinski, A 3D DLM/FD method for simulating the motion of spheres in a bounded shear flow of Oldroyd-B fluids, Computers \& Fluids 172 (2018) 661-673.

[42] T.-W. Pan, R. Glowinski, Numerical study of two disks settling in an Oldroyd-B fluid: From periodic interaction to chaining, Physical Review E 96 (2017) 063103.

[43] Y. Kim, M.-C. Lai, Y. Seol, A penalty immersed boundary method for viscoelastic particulate flows, Journal of Non-Newtonian Fluid Mechanics 258 (2018) 32-44.

[44] Y. Seol, Y.-H. Tseng, Y. Kim, M.-C. Lai, An immersed boundary method for simulating Newtonian vesicles in viscoelastic fluid, Journal of Computational Physics 376 (2019) 1009 1027.

[45] Y. Peng, L.-S. Luo, A comparative study of immersed-boundary and interpolated bounceback methods in LBE, Progress in Computational Fluid Dynamics 8 (2008) 156-167. 
[46] C. S. Peskin, Numerical analysis of blood flow in the heart, Journal of Computational Physics 25 (1977) 220-252.

[47] C. S. Peskin, The immersed boundary method, Acta Numerica 11 (2002) 479-517.

[48] S. Chen, G. D. Doolen, Lattice Boltzmann method for fluid flows, Annual Review of Fluid Mechanics 30 (1998) 329-364.

[49] C. K. Aidun, J. R. Clausen, Lattice-Boltzmann method for complex flows, Annual Review of Fluid Mechanics 42 (2010) 439-472.

[50] Y. Sui, Y. T. Chew, P. Roy, H. Low, A hybrid method to study flow-induced deformation of three-dimensional capsules, Journal of Computational Physics 227 (2008) 6351-6371.

[51] Z. Wang, Y. Sui, A.-V. Salsac, D. Barthès-Biesel, W. Wang, Motion of a spherical capsule in branched tube flow with finite inertia, Journal of Fluid Mechanics 806 (2016) 603-626.

[52] F.-B. Tian, H. Luo, L. Zhu, J. C. Liao, X.-Y. Lu, An efficient immersed boundary-lattice Boltzmann method for the hydrodynamic interaction of elastic filaments, Journal of Computational Physics 230 (2011) 7266-7283.

[53] F. B. Tian, H. Luo, X. Y. Lu, Coupling modes of three filaments in side-by-side arrangement, Physics of Fluids 23 (2011) 111903.

[54] F. B. Tian, H. Luo, L. Zhu, X. Y. Lu, Interaction between a flexible filament and a downstream rigid body, Physical Review E 82 (2010) 026301.

[55] F.-B. Tian, Role of mass on the stability of flag/flags in uniform flow, Applied Physics Letters 103 (2013) 034101.

[56] J. Ma, L. Xu, F.-B. Tian, J. Young, J. C. Lai, Dynamic characteristics of a deformable capsule in a simple shear flow, Physical Review E 99 (2019) 023101.

[57] F.-B. Tian, Deformation of a capsule in a power-law shear flow, Computational and Mathematical Methods in Medicine 2016 (2016) 7981386.

[58] D. Sun, Y. Wang, D. Jiang, N. Xiang, K. Chen, Z. Ni, Dynamic self-assembly of particles in an expanding channel flow, Applied Physics Letters 103 (2013) 071905.

[59] T. Krüger, B. Kaoui, J. Harting, Interplay of inertia and deformability on rheological properties of a suspension of capsules, Journal of Fluid Mechanics 751 (2014) 725-745.

[60] L. Xu, F.-B. Tian, J. Young, J. C. Lai, A novel geometry-adaptive cartesian grid based immersed boundary-lattice Boltzmann method for fluid-structure interactions at moderate and high Reynolds numbers, Journal of Computational Physics 375 (2018) 22-56.

[61] Y.-Q. Xu, M.-Y. Wang, Q.-Y. Liu, X.-Y. Tang, F.-B. Tian, External force-induced focus pattern of a flexible filament in a viscous fluid, Applied Mathematical Modelling 53 (2018) 369-383. 
[62] Z. Wang, Y. Sui, A.-V. Salsac, D. Barthès-Biesel, W. Wang, Path selection of a spherical capsule in a microfluidic branched channel: towards the design of an enrichment device, Journal of Fluid Mechanics 849 (2018) 136-162.

[63] D.-K. Sun, Z. Bo, Numerical simulation of hydrodynamic focusing of particles in straight channel flows with the immersed boundary-lattice Boltzmann method, International Journal of Heat and Mass Transfer 80 (2015) 139-149.

[64] D.-K. Sun, Y. Wang, A.-P. Dong, B.-D. Sun, A three-dimensional quantitative study on the hydrodynamic focusing of particles with the immersed boundary-lattice Boltzmann method, International Journal of Heat and Mass Transfer 94 (2016) 306-315.

[65] L. Zhu, X. Yu, N. Liu, Y. Cheng, X. Lu, A deformable plate interacting with a non-Newtonian fluid in three dimensions, Physics of Fluids 29 (2017) 083101.

[66] L. Zhu, A three-dimensional immersed boundary method for non-Newtonian fluids, Theoretical and Applied Mechanics Letters 8 (2018) 193-196.

[67] O. Malaspinas, N. Fiétier, M. Deville, Lattice Boltzmann method for the simulation of viscoelastic fluid flows, Journal of Non-Newtonian Fluid Mechanics 165 (2010) 1637-1653.

[68] Y. Sui, Y. Chew, P. Roy, X. Chen, H. Low, Transient deformation of elastic capsules in shear flow: effect of membrane bending stiffness, Physical Review E 75 (2007) 066301.

[69] D. J. Steigmann, R. W. Ogden, Plane deformations of elastic solids with intrinsic boundary elasticity, Proceedings of the Royal Society of London A: Mathematical, Physical and Engineering Sciences 453 (1997) 853-877.

[70] A. Guckenberger, M. P. Schraml, P. G. Chen, M. Leonetti, S. Gekle, On the bending algorithms for soft objects in flows, Computer Physics Communications 207 (2016) 1-23.

[71] R. Skalak, A. Tozeren, R. Zarda, S. Chien, Strain energy function of red blood cell membranes, Biophysical Journal 13 (1973) 245-264.

[72] W. Dodson III, P. Dimitrakopoulos, Tank-treading of erythrocytes in strong shear flows via a nonstiff cytoskeleton-based continuum computational modeling, Biophysical Journal 99 (2010) 2906-2916.

[73] W. Helfrich, Elastic properties of lipid bilayers: theory and possible experiments, Zeitschrift für Naturforschung C 28 (1973) 693-703.

[74] Z.-C. Ou-Yang, W. Helfrich, Bending energy of vesicle membranes: General expressions for the first, second, and third variation of the shape energy and applications to spheres and cylinders, Physical Review A 39 (1989) 5280.

[75] W. X. Huang, H. J. Sung, Three-dimensional simulation of a flapping flag in a uniform flow, Journal of Fluid Mechanics 653 (2010) 301-336.

[76] C. Denniston, E. Orlandini, J. Yeomans, Lattice Boltzmann simulations of liquid crystal hydrodynamics, Physical Review E 63 (2001) 056702. 
[77] D. Marenduzzo, E. Orlandini, M. Cates, J. Yeomans, Steady-state hydrodynamic instabilities of active liquid crystals: Hybrid lattice Boltzmann simulations, Physical Review E 76 (2007) 031921.

[78] D. Marenduzzo, E. Orlandini, M. Cates, J. Yeomans, Lattice Boltzmann simulations of spontaneous flow in active liquid crystals: The role of boundary conditions, Journal of NonNewtonian Fluid Mechanics 149 (2008) 56-62.

[79] J. Su, J. Ouyang, X. Wang, B. Yang, W. Zhou, Lattice Boltzmann method for the simulation of viscoelastic fluid flows over a large range of Weissenberg numbers, Journal of Non-Newtonian Fluid Mechanics 194 (2013) 42-59.

[80] B. E. Griffith, X. Luo, Hybrid finite difference/finite element immersed boundary method, International Journal for Numerical Methods in Biomedical Engineering 33 (2017) e2888.

[81] S. Ramanujan, C. Pozrikidis, Deformation of liquid capsules enclosed by elastic membranes in simple shear flow: large deformations and the effect of fluid viscosities, Journal of Fluid Mechanics 361 (1998) 117-143.

[82] T. Krüger, F. Varnik, D. Raabe, Efficient and accurate simulations of deformable particles immersed in a fluid using a combined immersed boundary lattice Boltzmann finite element method, Computers \& Mathematics with Applications 61 (2011) 3485-3505.

[83] A. Yazdani, P. Bagchi, Three-dimensional numerical simulation of vesicle dynamics using a front-tracking method, Physical Review E 85 (2012) 056308.

[84] X.-Q. Hu, A.-V. Salsac, D. Barthès-Biesel, Flow of a spherical capsule in a pore with circular or square cross-section, Journal of Fluid Mechanics 705 (2012) 176-194.

[85] J. Charrier, S. Shrivastava, R. Wu, Free and constrained inflation of elastic membranes in relation to thermoforming-non-axisymmetric problems, The Journal of Strain Analysis for Engineering Design 24 (1989) 55-74.

[86] S. Shrivastava, J. Tang, Large deformation finite element analysis of non-linear viscoelastic membranes with reference to thermoforming, The Journal of Strain Analysis for Engineering Design 28 (1993) 31-51.

[87] D. Matsunaga, Y. Imai, C. Wagner, T. Ishikawa, Reorientation of a single red blood cell during sedimentation, Journal of Fluid Mechanics 806 (2016) 102-128.

[88] M. Meyer, M. Desbrun, P. Schröder, A. H. Barr, Discrete differential-geometry operators for triangulated 2-manifolds, in: Visualization and mathematics III, Springer, 2003, pp. 35-57.

[89] W. X. Huang, S. J. Shin, H. J. Sung, Simulation of flexible filaments in a uniform flow by the immersed boundary method, Journal of Computational Physics 226 (2007) 2206-2228.

[90] L. Zhu, G. He, S. Wang, L. Miller, X. Zhang, Q. You, S. Fang, An immersed boundary method based on the lattice Boltzmann approach in three dimensions, with application, Computers \& Mathematics with Applications 61 (2011) 3506-3518. 
[91] W.-X. Huang, H. J. Sung, Three-dimensional simulation of a flapping flag in a uniform flow, Journal of Fluid Mechanics 653 (2010) 301-336.

[92] G. D'Avino, T. Tuccillo, P. Maffettone, F. Greco, M. Hulsen, Numerical simulations of particle migration in a viscoelastic fluid subjected to shear flow, Computers \& Fluids 39 (2010) 709-721.

[93] Z. Gou, F. Huang, X. Ruan, X. Fu, Shape memory of elastic capsules under the effect of spontaneous shape, Communications in Computational Physics 24 (2018) 234- 252.

[94] W. X. Huang, C. B. Chang, H. J. Sung, Three-dimensional simulation of elastic capsules in shear flow by the penalty immersed boundary method, Journal of Computational Physics 231 (2012) 3340-3364.

[95] H. Zhao, E. S. Shaqfeh, The dynamics of a vesicle in simple shear flow, Journal of Fluid Mechanics 674 (2011) 578-604.

[96] M. Kraus, W. Wintz, U. Seifert, R. Lipowsky, Fluid vesicles in shear flow, Physical Review Letters 77 (1996) 3685.

[97] N. J. Zabusky, E. Segre, J. Deschamps, V. Kantsler, V. Steinberg, Dynamics of vesicles in shear and rotational flows: Modal dynamics and phase diagram, Physics of Fluids 23 (2011) 041905 .

[98] H. Liu, J. Zhou, R. Burrows, Inlet and outlet boundary conditions for the lattice-Boltzmann modelling of shallow water flows, Progress in Computational Fluid Dynamics, an International Journal 12 (2012) 11-18.

[99] T. Krüger, H. Kusumaatmaja, A. Kuzmin, O. Shardt, G. Silva, E. M. Viggen, Lattice Boltzmann for advection-diffusion problems, in: The Lattice Boltzmann Method, Springer, 2017, pp. 297-329.

[100] E. Ezzatneshan, Implementation of D3Q19 lattice B"oltzmann method with a curved wall boundary condition for simulation of practical flow problems, International Journal of Engineering 30 (2017) 1381-1390.

[101] F.-B. Tian, H. Dai, H. Luo, J. F. Doyle, B. Rousseau, Fluid-structure interaction involving large deformations: 3D simulations and applications to biological systems, Journal of Computational Physics 258 (2014) 451-469.

[102] M. D. de Tullio, G. Pascazio, A moving-least-squares immersed boundary method for simulating the fluid-structure interaction of elastic bodies with arbitrary thickness, Journal of Computational Physics 325 (2016) 201-225.

[103] I. Lee, H. Choi, A discrete-forcing immersed boundary method for the fluid-structure interaction of an elastic slender body, Journal of Computational Physics 280 (2015) 529-546.

[104] A. H. Raffiee, S. Dabiri, A. M. Ardekani, Deformation and buckling of microcapsules in a viscoelastic matrix, Physical Review E 96 (2017) 032603.

[105] J. C. R. Hunt, A. Wray, P. Moin, Eddies, stream, and convergence zones in turbulent flows, Center for Turbulence Research Report CTR-S88, 1988. 